\title{
Spectral pollution and second order relative spectra for self-adjoint operators
}

\author{
Michael Levitin \\ Department of Mathematics, Heriot-Watt University, \\ Riccarton, Edinburgh EH14 4AS \\ M.Levitin@ma.hw.ac.uk \\ www.ma.hw.ac.uk/ levitin \\ Eugene Shargorodsky \\ School of Mathematical Sciences, University of Sussex, \\ Falmer, Brighton, BN1 9QH, UK \\ E.Shargorodsky@sussex.ac.uk \\ www.maths.sussex.ac.uk/Staff/ES/
}

October 30, 2018

\begin{abstract}
We consider the phenomenon of spectral pollution arising in calculation of spectra of self-adjoint operators by projection methods. We suggest a strategy of dealing with spectral pollution by using the socalled second order relative spectra. The effectiveness of the method is illustrated by a detailed analysis of two model examples.
\end{abstract}

Keywords: computation of eigenvalues, spectral pollution, projection methods, spurious eigenvalues, Agmon-Douglis-Nirenberg elliptic operators

2000 Mathematics Subject Classification: 47A75, 65J10 


\section{Contents}

1 Introduction 4

\begin{tabular}{lll}
\hline 2 & Main results & 7
\end{tabular}

3 Example I: operator of multiplication by a discontinuous $\begin{array}{lr}\text { function } & 9\end{array}$

4 Example II: Stokes type system of ODEs 16

\begin{tabular}{|ll}
5 Proofs of the main results & 21
\end{tabular}

6 More on Example 1 25

\begin{tabular}{lll}
\hline 7 & More on Example II & 29
\end{tabular}

\begin{tabular}{|lll}
\hline & Concluding remarks & 34
\end{tabular}

\begin{tabular}{ll}
\hline Acknowledgements & 35
\end{tabular}

\begin{tabular}{ll}
\hline References & 35
\end{tabular}

\section{List of Figures}

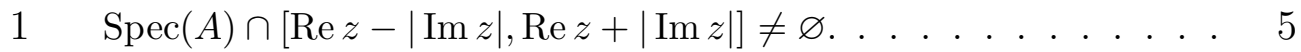

$2 \quad \operatorname{Spec}\left(A, \mathcal{L}_{1001}\right) . \ldots \ldots \ldots \ldots$

3 A typical computed "eigenfunction" of $A$ corresponding to a spurious eigenvalue. . . . . . . . . . . . . . . 11

$4 \quad \operatorname{Spec}_{2}\left(A, \mathcal{L}_{N}\right) . \ldots \ldots \ldots \ldots$

$5 \quad$ Detailed view of Figure 4 near the real axis. . . . . . . . . . . . . 13

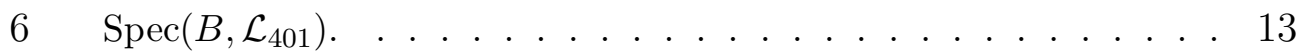

$7 \quad \operatorname{Spec}_{2}\left(B, \mathcal{L}_{N}\right) . \ldots \ldots \ldots \ldots$

$8 \quad$ Detailed view of Figure 7 near the real axis and, for comparison, $\operatorname{Spec}\left(B, \mathcal{L}_{N}\right) \ldots \ldots \ldots \ldots \ldots \ldots$

$9 \quad$ FEM calculated $\operatorname{Spec}\left(H, \mathbf{L}_{N} \times \mathbf{L}_{N}\right)$ plotted against $N$. All eigenvalues in the interval $(1,2)$ are spurious! . . . . . . . . . . . . . . 18

$10 \quad$ Second order relative spectra $\operatorname{Spec}_{2}\left(H, \mathbf{M}_{N} \times \mathbf{L}_{N}\right)$ for various values of $N$ - low eigenvalues. . . . . . . . . . . . . . . . . . . . . . . 19

11 Second order relative spectra $\operatorname{Spec}_{2}\left(H, \mathbf{M}_{N} \times \mathbf{L}_{N}\right)$ for various values of $N-$ all eigenvalues. . . . . . . . . . . . . . . . . . . . . . . . 20

$12 \quad \operatorname{Spec}\left(A, \mathcal{L}_{1000}\right) . \ldots \ldots \ldots \ldots$ 
13 Points of $\operatorname{Spec}\left(A_{y}, \mathcal{L}_{N}\right), y=-\pi / 3$, in the interval $(-1,1)$ as functions of $N$. . . . . . . . . . . . . . . . . . . . . . 27

14 Points of $\operatorname{Spec}\left(B_{y}, \mathcal{L}_{N}\right), y=-\pi / 3$, in the interval $(-1,1)$ as functions of $N$. Note the presence of both "genuine" and "spurious" eigenvalues. . . . . . . . . . . . . . . . . . 28

$15 \quad$ Points of $\operatorname{Spec}\left(A_{y}, \mathcal{L}_{N}\right)$ in the interval $(-1,1)$ as functions of $N$, plotted for various values of $y . \ldots . \ldots . \ldots 29$

$16 \quad$ FEM calculated $\operatorname{Spec}\left(H, \mathbf{M}_{N} \times \mathbf{L}_{N}\right)$ plotted against $N$. There are no spurious eigenvalues! . . . . . . . . . . . . . . . . 30

$17 \operatorname{Spec}\left(H, \mathbf{M}_{N} \times \mathbf{M}_{N}\right)$ plotted against $N$. Again, all the eigenvalues in the interval $(1,2)$ are spurious! . . . . . . . . . . . . . . . 31

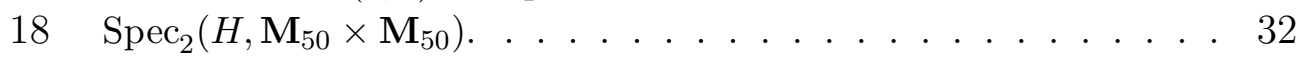

\section{List of Tables}

$1 \quad$ Eigenvalues lying in the gap of the essential spectrum of $A$ for some values of $N$. . . . . . . . . . . . . . . . . . . . . . . . . . . . 10

$2 \quad$ Eigenvalues lying in the gap of the essential spectrum of $A$ for some even values of $N . \ldots \ldots \ldots$

$3 \quad$ Eigenvalues lying in the gap of the essential spectrum of $A$ for some consecutive values of $N .2$-periodicity in $N$ is clearly visible. . . . 27 


\section{Introduction}

Let $A$ be a self-adjoint operator on a Hilbert space $\mathcal{H}$. In order to determine the spectrum $\operatorname{Spec}(A)$ of $A$, one can use the following projection method. Let $\left(\mathcal{L}_{k}\right)_{k \in \mathbb{N}}$ be a sequence of closed linear subspaces of the domain $\operatorname{Dom}(A)$ of $A$ and suppose that the corresponding orthogonal projections $P_{k}: \mathcal{H} \rightarrow \mathcal{L}_{k}$ converge strongly to the identity operator $I$. Let $\Lambda(A)$ be the set of all such sequences of subspaces. One may ask whether or not

$$
\lim _{k \rightarrow \infty} \operatorname{Spec}\left(A, \mathcal{L}_{k}\right)=\operatorname{Spec}(A)
$$

where $\operatorname{Spec}\left(A, \mathcal{L}_{k}\right)$ is the spectrum of $P_{k} A: \mathcal{L}_{k} \rightarrow \mathcal{L}_{k}$ and "lim" is defined in an appropriate sense. Unfortunately the answer is, in general, negative and $\lim _{k \rightarrow \infty} \operatorname{Spec}\left(A, \mathcal{L}_{k}\right)$ may contain points which do not belong to $\operatorname{Spec}(A)$. This phenomenon is well known in numerical analysis and is called spectral pollution.

Since we allow $A$ to be unbounded, it is convenient for us to extend $\operatorname{Spec}(A)$ by adding to it $-\infty,+\infty$, or both, if $A$ is unbounded below, above or from both sides, respectively. We denote the resulting set by $\widehat{\operatorname{Spec}}(A)$. In other words, $\widehat{\operatorname{Spec}}(A)$ is the closure of $\operatorname{Spec}(A)$ in the two point compactification $\overline{\mathbb{R}}:=\mathbb{R} \cup\{ \pm \infty\}$ of $\mathbb{R}$. Let $\operatorname{Spec}_{\text {ess }}(A)$ be the essential spectrum of $A$, i.e.

$\operatorname{Spec}_{\text {ess }}(A):=\operatorname{Spec}(A) \backslash\{$ isolated eigenvalues of finite multiplicity $\}$,

and let $\widehat{S}_{\text {pec }}(A)$ be the extended essential spectrum of $A$, i.e.

$$
\widehat{\operatorname{Spec}}_{\mathrm{ess}}(A):=\widehat{\operatorname{Spec}}(A) \backslash\{\text { isolated eigenvalues of finite multiplicity }\} \text {. }
$$

It turns out that spectral pollution may occur at any point lying in a gap of the extended essential spectrum, i.e. at any point of the set $\operatorname{conv}\left(\widehat{\operatorname{Spec}}_{\mathrm{ess}}(A)\right) \backslash \widehat{\mathrm{Spec}}_{\mathrm{ess}}(A)$, where "conv" means the convex hull (see the proof of Theorem 2.1 below, where this fact is demonstrated by choosing an appropriate sequence of finite-dimensional subspaces).

One can argue that the sequence $\left(\mathcal{L}_{k}\right)_{k \in \mathbb{N}}$ in the proof of Theorem 2.1 had been deliberately chosen in a particularly nasty way and that things like this do not usually happen in "real life". This argument is only partially true at best, and examples in Sections 3 and 4 show how the standard approach leads to spectral pollution in some very straightforward problems.

One can try to deal with spectral pollution by choosing $\mathcal{L}_{k}$ in a "reasonable way". However the known recipes (see, e.g., [BBG], [BDG], [RSHSPV], 
and references therein) are by no means universal or rigorously justified for a wide range of problems. An absolutely "safe" choice of $\left(\mathcal{L}_{k}\right)_{k \in \mathbb{N}}$ would be the one based on the spectral decomposition of $A$. Unfortunately knowing the spectral decomposition is much more than knowing just the spectrum which is to be found.

We propose a complementary (not an alternative!) way of dealing with spectral pollution, which is based on the notion of the second order spectrum $\operatorname{Spec}_{2}(A, \mathcal{L})$ of $A$ relative to a subspace $\mathcal{L} \subset \operatorname{Dom}(A)$ (see Definition 2.3) introduced in Dav. Second order relative spectra usually contain complex numbers, but it turns out (see Theorem 2.5) that if $z \in \operatorname{Spec}_{2}(A, \mathcal{L})$ then

$$
\operatorname{Spec}(A) \cap[\operatorname{Re} z-|\operatorname{Im} z|, \operatorname{Re} z+|\operatorname{Im} z|] \neq \varnothing \operatorname{~}
$$

In particular, this means that if $z$ belongs to a second order relative spectrum of $A$, and its imaginary part is small, than there is a point of the spectrum of $A$ close to Re $z$.

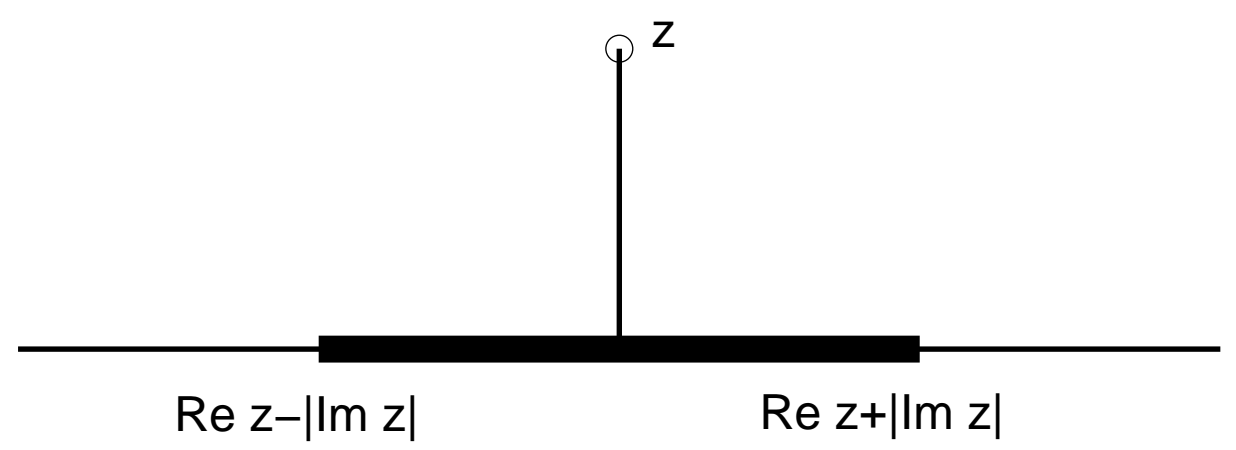

Figure 1: $\operatorname{Spec}(A) \cap[\operatorname{Re} z-|\operatorname{Im} z|, \operatorname{Re} z+|\operatorname{Im} z|] \neq \varnothing$.

This fact suggests the following strategy, which uses (2) as a posteriori estimate. One should choose a reasonable sequence $\left(\mathcal{L}_{k}\right)_{k \in \mathbb{N}}$ and apply the standard projection method, i.e. find $\operatorname{Spec}\left(A, \mathcal{L}_{k}\right)$. Additionally, one should find $\operatorname{Spec}_{2}\left(A, \tilde{\mathcal{L}}_{k}\right)$ (for possibly different $\left.\tilde{\mathcal{L}}_{k} \subset \operatorname{Dom}(A)\right)$. Suppose $\operatorname{Spec}\left(A, \mathcal{L}_{k}\right)$ contain points which appear to converge to some $\lambda \in \mathbb{R}$ as $k \rightarrow \infty$. If $\operatorname{Spec}_{2}\left(A, \tilde{\mathcal{L}}_{k}\right)$ also contain points lying close to $\lambda$, then $\lambda$ is indeed close to the spectrum of $A$. If however $\operatorname{Spec}_{2}\left(A, \tilde{\mathcal{L}}_{k}\right)$ do not contain points approximating $\lambda$, one cannot conclude that $\lambda$ is far from the spectrum. A definite claim which can be made in this case is that $\lambda$ requires additional attention円, as

\footnotetext{
${ }^{1}$ One can, for example, use the method suggested in the companion paper by E.B. Davies and M. Plum DP].
} 
spectral pollution may be around the corner. It has to be said though, that our (very limited!) experience seems to suggest that $\operatorname{Spec}_{2}\left(A, \tilde{\mathcal{L}}_{k}\right)$ do capture the spectrum quite well.

We do not claim that the above method is going to be universally successful. Our main ambition is merely to bring it to the attention of those concerned with finding spectra numerically. We have applied it to two problems which were chosen mainly for their simplicity. The results (which turned out to be better than we expected) are presented in Sections 3, 4, 6, and 7 .

Section 2 contains the main theoretical results on standard (first order) and second order relative spectra. Similar results on second (and higher) order relative spectra have been obtained in Sha] under slightly stronger restrictions. We start with Theorem 2.1, which has been mentioned above and which says that spectral pollution may occur at any point lying in a gap of the extended essential spectrum. It implies that one should be particularly careful when applying a projection method to find the spectrum of an operator $A$ in the following cases:

a) $A$ is not semi-bounded and $\operatorname{Spec}(A) \neq \mathbb{R}$;

b) $A$ is semi-bounded but unbounded, and has a nonempty essential spectrum different from a half-line;

c) $A$ is bounded and has at least one gap in the essential spectrum.

Examples of types c) and b) are treated in Sections 3 and 4 respectively. We return to these examples in Sections 6 and 7 after giving the proofs of the main theoretical results in Section 5.

Section 2 also discusses an additional difficulty which arises for unbounded operators. It is well known that if $A$ is bounded, then the left-hand side of (11) contains the right-hand side. If, however, $A$ is unbounded above, say, then the left-hand side of (11) may well be equal to $\{+\infty\}$ (see Theorem 2.2).

In order to make the paper more readable, we have divided the material into two parts. The first one consists of Sections 2-4 and covers the main theoretical and numerical results. The same material is treated at a greater depth in the second part of the paper, which consists of Sections 5-7. The summary is given in Section 8.

Finally, the accompanying webpage

www.ma.hw.ac.uk/ levitin/SpectralPollution

contains the listings of the Matlab programmes written by the authors and used for numerical calculations in Examples I and II. 


\section{Main results}

Let $A$ be a self-adjoint operator on a Hilbert space $\mathcal{H}$. Throughout the paper $E(\cdot)$ denote the spectral projections associated with $A$. The proofs of all results of this Section are given in Section 5 .

The following result states that spectral pollution may occur at any point in a gap of the extended essential spectrum of $A$.

Theorem 2.1. For any $\lambda \in \operatorname{conv}\left(\widehat{\operatorname{Spec}}_{\mathrm{ess}}(A)\right) \backslash \widehat{\operatorname{Spec}}_{\mathrm{ess}}(A)$ there exists an increasing sequence $\left(\mathcal{L}_{k}\right)_{k \in \mathbb{N}} \in \Lambda(A)$ such that

$$
\lambda \in \operatorname{Spec}\left(A, \mathcal{L}_{k}\right), \quad \forall k \in \mathbb{N} .
$$

It is well known that for bounded self-adjoint operators the left-hand side of (四) contains the right-hand side:

$$
\lim _{k \rightarrow \infty} \operatorname{Spec}\left(A, \mathcal{L}_{k}\right) \supseteq \operatorname{Spec}(A)
$$

(see, e.g., Arv or [Sha]). So, spectral pollution means that the left-hand side of (1) is strictly larger than the right-hand side. For unbounded self-adjoint operators the situation is more complicated: the left-hand side of (1) does not necessarily contain the right-hand side. The reason for this is intuitively very clear. Let $\mathcal{L}_{k}$ be finite dimensional and suppose for definiteness that $A$ is not bounded above. Then perturbing a basis of $\mathcal{L}_{k}$ by arbitrarily small vectors lying in $\operatorname{Dom}(A) \cap E([N,+\infty)) \mathcal{H}$ with sufficiently large $N$, one can push the spectrum of the corresponding finite dimensional operator arbitrarily far to the right.

Theorem 2.2. Let $A$ be an unbounded above self-adjoint operator on a separable Hilbert space $\mathcal{H}$. Then for an arbitrary sequence $\left(\mathcal{L}_{k}\right)_{k \in \mathbb{N}} \in \Lambda(A)$ of finite dimensional subspaces and arbitrary sequences $\left(\varepsilon_{k}\right)_{k \in \mathbb{N}} \searrow 0$ and $\left(R_{k}\right)_{k \in \mathbb{N}} \nearrow+\infty$, there exists a sequence $\left(\mathcal{L}_{k}^{\prime}\right)_{k \in \mathbb{N}} \in \Lambda(A)$ such that

$$
\left\|P_{k}-P_{k}^{\prime}\right\|<\varepsilon_{k} \quad \text { and } \quad \operatorname{Spec}\left(A, \mathcal{L}_{k}^{\prime}\right) \subset\left(R_{k},+\infty\right), \quad \forall k \in \mathbb{N},
$$

where $P_{k}^{\prime}: \mathcal{H} \rightarrow \mathcal{L}_{k}^{\prime}$ are the corresponding orthogonal projections. A similar statement holds for operators unbounded below.

The requirement $\mathcal{L}_{k} \subset \operatorname{Dom}(A)$ is too restrictive for many applications, in particular for approximating spectra of differential operators by finite element methods. When using finite element methods, one usually states the problem in a weak (variational) form and requires that $\mathcal{L}_{k}$ belong to the domain of 
the corresponding sesquilinear form. The restriction $\mathcal{L}_{k} \subset \operatorname{Dom}(A)$ would mean extra smoothness of the finite elements.

Suppose $A$ is a bounded below self-adjoint operator on a separable Hilbert space $\mathcal{H}$ and let $Q_{A}: \mathcal{H} \times \mathcal{H} \rightarrow \mathbb{C}$ be the corresponding closed sesquilinear form (see, e.g., [Kat, $\S 1$, Ch. VI]). Let $\left(\mathcal{L}_{k}\right)_{k \in \mathbb{N}}$ be a sequence of finite dimensional linear subspaces of the domain $\operatorname{Dom}\left(Q_{A}\right)$ of $Q_{A}$ such that the corresponding orthogonal projections $P_{k}: \mathcal{H} \rightarrow \mathcal{L}_{k}$ converge strongly to $I$. Let $\Lambda\left(Q_{A}\right)$ be the set of all such sequences. A number $\lambda \in \mathbb{C}$ is said to belong to $\operatorname{Spec}\left(A, \mathcal{L}_{k}\right)$ if there exists $u \in \mathcal{L}_{k} \backslash\{0\}$ such that

$$
Q_{A}(u, v)-\lambda(u, v)=0, \quad \forall v \in \mathcal{L}_{k}
$$

If $\mathcal{L}_{k} \subset \operatorname{Dom}(A)$, then $Q_{A}(u, v)=(A u, v)$ and the above definition means that

- there exists $u \in \mathcal{L}_{k} \backslash\{0\}$ such that $(A u, v)-\lambda(u, v)=0, \forall v \in \mathcal{L}_{k}$,

- i.e. there exists $u \in \mathcal{L}_{k} \backslash\{0\}$ such that $P_{k} A u=\lambda u$

- i.e. $\lambda$ belongs to the spectrum of $P_{k} A: \mathcal{L}_{k} \rightarrow \mathcal{L}_{k}$.

Hence one gets the old definition of $\operatorname{Spec}\left(A, \mathcal{L}_{k}\right)$ for finite dimensional $\mathcal{L}_{k} \subset$ $\operatorname{Dom}(A)$ (cf. Remarks 2.4 and 2.6(b) below).

It is not difficult to prove that Theorem 2.2 remains true if $\Lambda(A)$ is replaced by $\Lambda\left(Q_{A}\right)$ (see Section 5$)$.

The above results show the difficulties one may face when trying to approximate the spectrum of a self-adjoint operator with the help of a projection method. A possible way of addressing spectral pollution is based on the notion of the second order relative spectrum.

Definition 2.3. Let $A$ be a self-adjoint operator on a Hilbert space $\mathcal{H}$ and let $\mathcal{L}$ be a finite dimensional subspace of $\operatorname{Dom}(A)$. A number $z \in \mathbb{C}$ is said to belong to the second order spectrum $\operatorname{Spec}_{2}(A, \mathcal{L})$ of $A$ relative to $\mathcal{L}$ if there exists $u \in \mathcal{L} \backslash\{0\}$ such that

$$
((A-z I) u,(A-\bar{z} I) v)=0, \quad \forall v \in \mathcal{L}
$$

where $(\cdot, \cdot)$ is the scalar product in $\mathcal{H}$.

Remark 2.4. Suppose $\mathcal{L} \subset \operatorname{Dom}\left(A^{2}\right)$ and let $P$ be the orthogonal projection onto $\mathcal{L}$. Then $z \in \operatorname{Spec}_{2}(A, \mathcal{L})$

- iff there exists $u \in \mathcal{L} \backslash\{0\}$ such that $\left((A-z I)^{2} u, v\right)=0, \forall v \in \mathcal{L}$,

- i.e. iff there exists $u \in \mathcal{L} \backslash\{0\}$ such that $u \in \operatorname{Ker}\left(P(A-z I)^{2}\right)$,

- i.e. iff the operator $P(A-z I)^{2}: \mathcal{L} \rightarrow \mathcal{L}$ is not invertible.

The last is the definition of the second order relative spectrum introduced in Dav and used in Sha. 
Theorem 2.5. If $z \in \operatorname{Spec}_{2}(A, \mathcal{L})$ then

$$
\operatorname{Spec}(A) \cap[\operatorname{Re} z-|\operatorname{Im} z|, \operatorname{Re} z+|\operatorname{Im} z|] \neq \varnothing .
$$

Remark 2.6. (a) Theorem 2.5 has been proved in Sha in the case $\mathcal{L} \subset$ $\operatorname{Dom}\left(A^{2}\right)$.

(b) Everything said above about second order relative spectra remains unchanged if $\mathcal{L}$ is infinite dimensional, with only one exception: the implication

$$
P(A-z I)^{2}: \mathcal{L} \rightarrow \mathcal{L} \text { is not invertible } \Longrightarrow \operatorname{Ker}\left(P(A-z I)^{2}\right) \cap \mathcal{L} \neq\{0\}
$$

(see Remark 2.4) is no longer true.

\section{Example I: operator of multiplication by a discontinuous function}

Consider the operator of multiplication by a function $a$ :

$$
A=a I: L_{2}([-\pi, \pi]) \rightarrow L_{2}([-\pi, \pi]),
$$

where

$$
a(x)= \begin{cases}-\frac{3}{2}+\frac{1}{2} \cos \sqrt{5} x, & \text { for } \quad-\pi \leq x<0, \\ 2+\cos \sqrt{2} x, & \text { for } \quad 0 \leq x<\pi .\end{cases}
$$

It is clear that

$$
\operatorname{Spec}(A)=\operatorname{Spec}_{\mathrm{ess}}(A)=a([-\pi, \pi])=[-2,-1] \cup[1,3] .
$$

The function $a$ and, in particular, the irrational factors $\sqrt{5}$ and $\sqrt{2}$ have been chosen to get a "generic" situation and avoid any possible hidden symmetries.

Using the standard orthonormal basis $e_{k}(x):=(2 \pi)^{-1 / 2} \exp (i k x), k \in \mathbb{Z}$, one can represent $A$ as a (doubly) infinite Toeplitz matrix and take the orthogonal projections onto $\mathcal{L}_{N}:=\operatorname{span}\left(\left\{e_{k}\right\}_{k=-n}^{n}\right)$, where $N=\operatorname{dim} \mathcal{L}_{N}=$ $2 n+1$. Figure 2 shows the approximation of the spectrum obtained by the standard projection method for a typical value of $N$. One can see that the essential spectrum $[-2,-1] \cup[1,3]$ is approximated very well, but that, additionally, there are some computed eigenvalues lying in the gap of the essential spectrum.

The spurious eigenvalues vary very slowly with $N$, see Table 1, and their number \# $\left(\operatorname{Spec}\left(A, \mathcal{L}_{N}\right) \cap(-1,1)\right)$ also increases slowly as $N$ increases. In fact, they seem to converge monotonically to some points in $(-1,1)$, and 


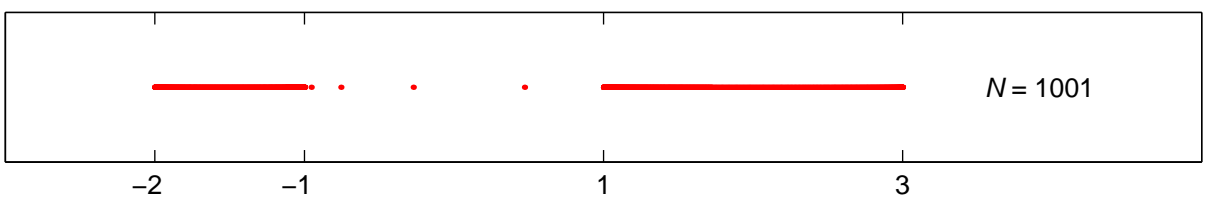

Figure 2: $\operatorname{Spec}\left(A, \mathcal{L}_{1001}\right)$.

\begin{tabular}{|c|rrrr|}
\hline$N$ & 101 & 401 & 701 & 1001 \\
\hline & & & -0.9997 & -0.9985 \\
$\operatorname{Spec}\left(A, \mathcal{L}_{N}\right) \cap(-1,1)$ & & -0.9834 & -0.9673 & -0.9550 \\
& -0.9264 & -0.8250 & -0.7823 & -0.7553 \\
& -0.4866 & -0.3478 & -0.3002 & -0.2721 \\
& 0.4362 & 0.4597 & 0.4673 & 0.4717 \\
\hline
\end{tabular}

Table 1: Eigenvalues lying in the gap of the essential spectrum of $A$ for some values of $N$.

judging by the numerical evidence alone, one might have thought that the limits belonged to the spectrum, if the example was less trivial and the spectrum was not known.

The presence of spurious points is hardly surprising. Indeed, if instead of the operator of multiplication by $a$, one considers the corresponding Toeplitz operator $T_{a}$ acting on the Hardy space $\mathrm{H}^{2}=\operatorname{span}\left(\left\{e_{k}\right\}_{k \geq 0}\right)$, then the projection method using $\mathcal{M}_{N}:=\operatorname{span}\left(\left\{e_{k}\right\}_{k=0}^{N-1}\right)$ leads to the same finite-dimensional Toeplitz matrices. In other words, the standard projection method does not know which spectrum is being calculated: that of the operator of multiplication or of the Toeplitz operator. The latter equals $[-2,3]$ (see [HW] or [BS, 2.36]). Therefore not only will we have spectral pollution, the spurious eigenvalues will eventually fill the entire gap $(-1,1)$ (cf. (3) or [Böt).

Remark 3.1. It is interesting that spurious eigenvalues appear so slowly. Three spurious eigenvalues are present already for rather small values of $N$. The fourth one emerges from -1 for $N \approx 161$, and the fifth one emerges from -1 for $N \approx 631$. Such a slow rate seems to have the following explanation. The part $(-1,1)$ of the spectrum of the Toeplitz operator $T_{a}$ is there because of the discontinuities of $a(x)$ at $x=0$ and $x=-\pi \sim \pi$. Functions responsible for this part of the spectrum appear to be very localized at 0 and $-\pi \sim \pi$ and hence have a very slow decay of the Fourier coefficients. Figure 3 shows the graph of the trigonometric polynomial which is an eigenfunction of $P_{N} A$ : $\mathcal{L}_{N} \rightarrow \mathcal{L}_{N}, N=1001$, corresponding to $\lambda \approx 0.4717$. 


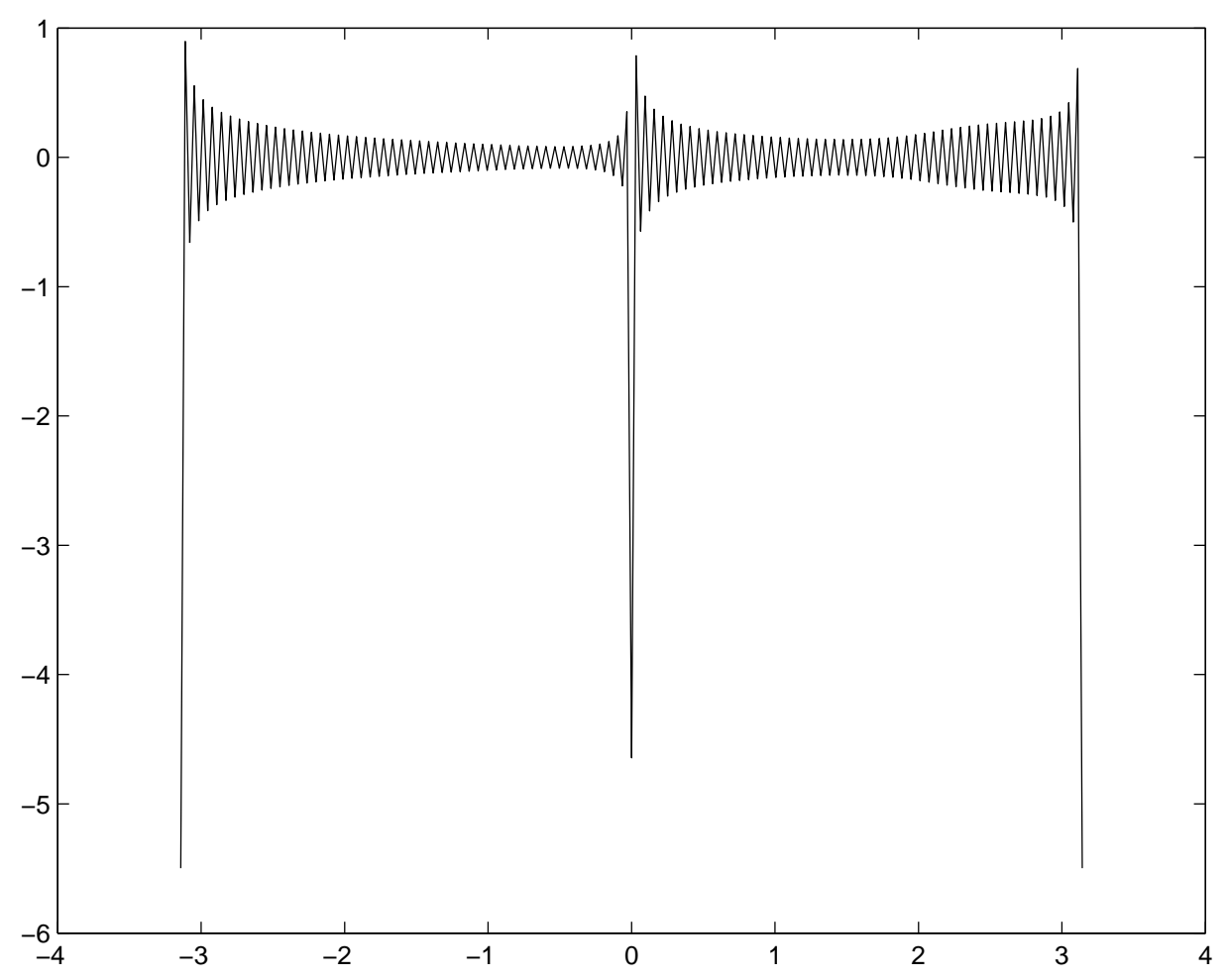

Figure 3: A typical computed "eigenfunction" of $A$ corresponding to a spurious eigenvalue.

We now show how second order relative spectra locate the spectrum of $A$ and, in particular, detect spectral pollution in the standard projection method.

Figure 1 shows the second order relative spectra $\operatorname{Spec}_{2}\left(A, \mathcal{L}_{N}\right)$ computed for $N=101,201,501$.

For each $N$, the second order relative spectrum consists of a few points lying approximately on a circle centred on the real axis and two contours "surrounding" two intervals of the (essential) spectrum. The number of points of $\operatorname{Spec}_{2}\left(A, \mathcal{L}_{N}\right)$ lying on the circle does not seem to increase with $N$.

Figure 5 gives a detailed view of Figure 1 near the real axis (note the different scales along the coordinate axes). The contours made of the points of the second order relative spectra, which "surround" two intervals of the (essential) spectrum, get closer to the real line as $N$ increases. Most importantly, the absence of points of the second order relative spectra close to the eigenvalues found by the standard projection method inside the interval $(-1,1)$ suggests that these eigenvalues are spurious. 


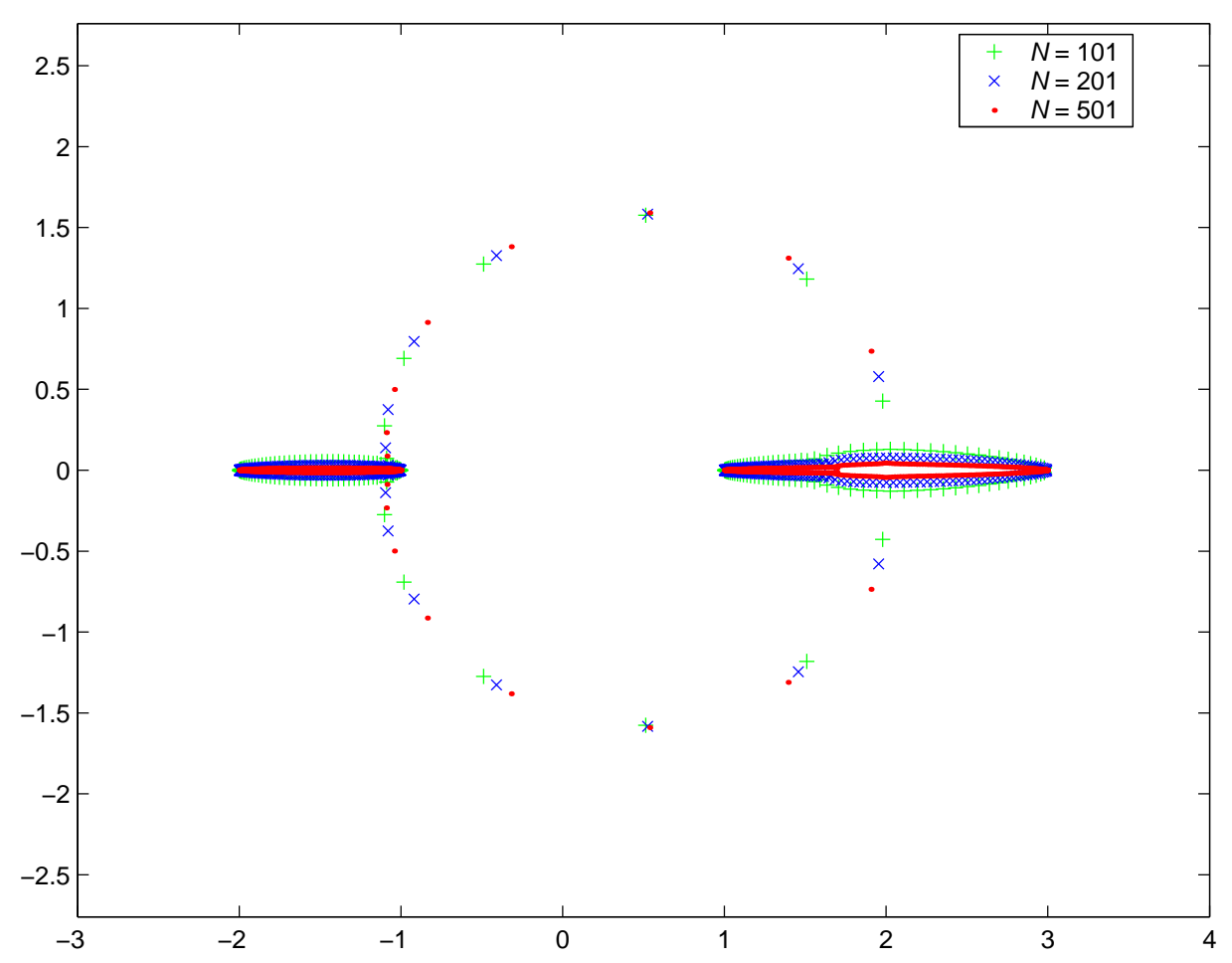

Figure 4: $\operatorname{Spec}_{2}\left(A, \mathcal{L}_{N}\right)$.

In order to illustrate a situation when an operator has both non-empty essential and discrete spectra, let us consider a two-dimensional perturbation of $A=a I$,

$$
\begin{aligned}
B u(x):=a(x) u(x) & -\frac{3 \exp (-i x)}{2 \pi} \int_{-\pi}^{\pi} u(t) \exp (i t) d t \\
& +\frac{\exp (2 i x)}{2 \pi} \int_{-\pi}^{\pi} u(t) \exp (-2 i t) d t, \quad x \in[-\pi, \pi] .
\end{aligned}
$$

This time, the essential spectrum is again very well approximated by the usual projection method, and the perturbation gives rise to several eigenvalues, see Figure 6, listed here for $N=401:$ one $\left(\lambda_{1} \approx-3.6057\right)$ to the left of the essential spectrum, six $\left(\lambda_{2} \approx-0.9842, \lambda_{3} \approx-0.8947, \lambda_{4} \approx-0.8203\right.$, $\lambda_{5} \approx-0.3496, \lambda_{6} \approx 0.4603$, and $\left.\lambda_{7} \approx 0.6082\right)$ in the gap $(-1,1)$, and one $\lambda_{8} \approx 3.0432$ ) to the right of the essential spectrum.

To establish which of the eight eigenvalues are spurious, we compute the second order relative spectra of $B$. Their structure is similar to that of $\operatorname{Spec}_{2}\left(A, \mathcal{L}_{N}\right)$ apart from several additional points located very close to the 


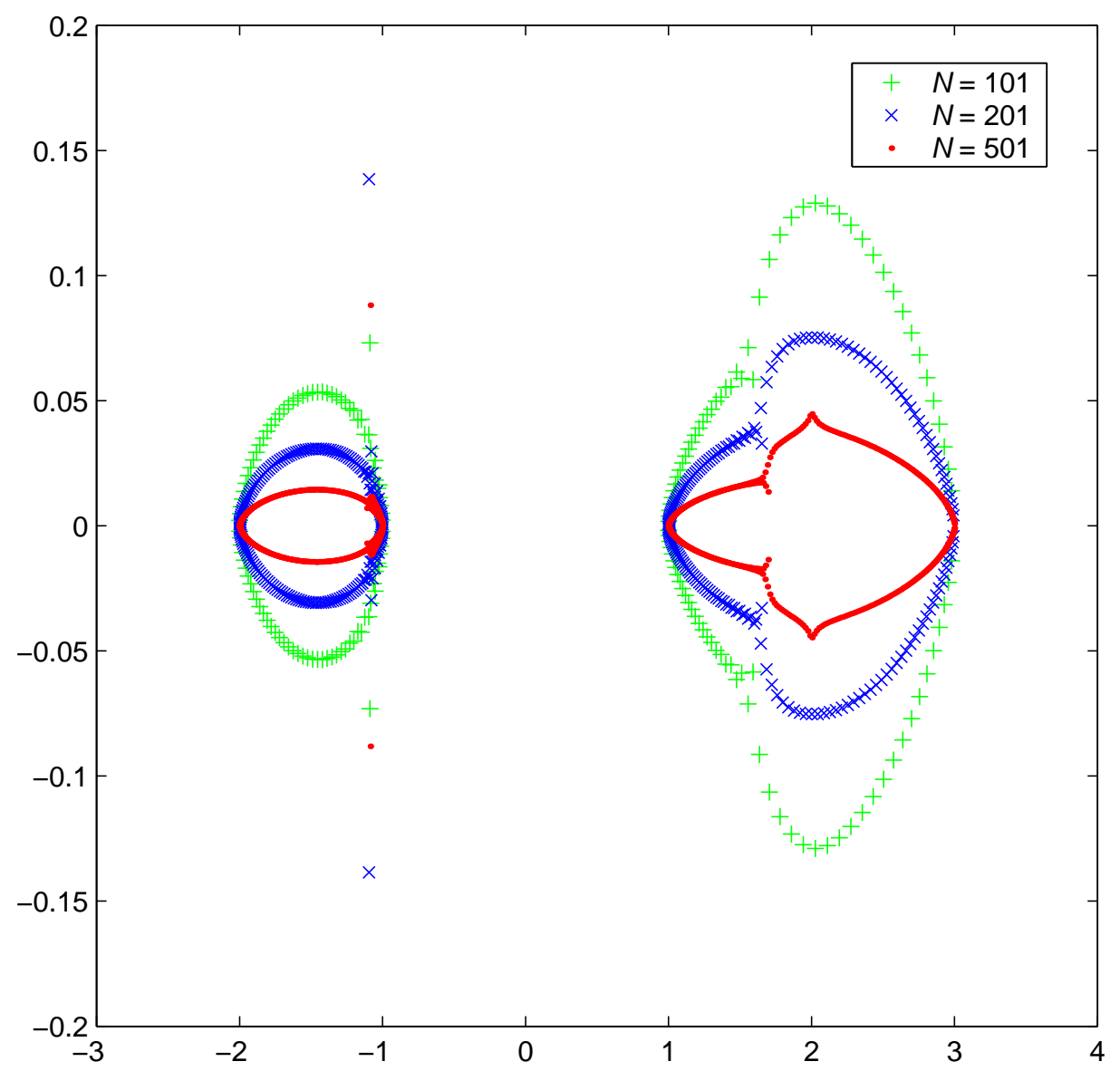

Figure 5: Detailed view of Figure 4 near the real axis.

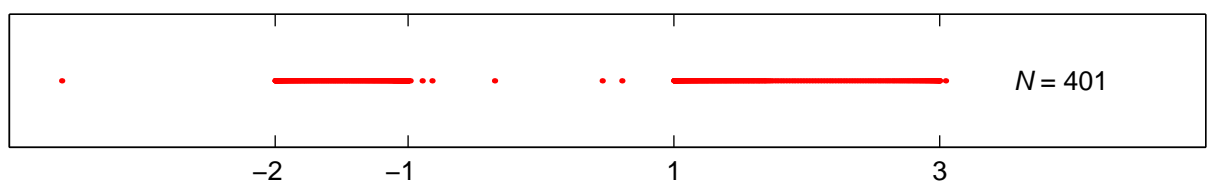

Figure 6: $\operatorname{Spec}\left(B, \mathcal{L}_{401}\right)$.

real axis, see Figures 7 and 8. In particular, for $N=401$ these points are approximately $-3.6056 \pm 0.0505 i,-0.8929 \pm 0.0266 i, 0.6085 \pm 0.0442 i$, and $3.0413 \pm 0.0178 i$. Thus, the eigenvalues $\lambda_{1}, \lambda_{3}, \lambda_{7}$ and $\lambda_{8}$ are correct, whereas the other eigenvalues $\lambda_{2}, \lambda_{4}, \lambda_{5}$, and $\lambda_{6}$ are almost certainly spurious. 


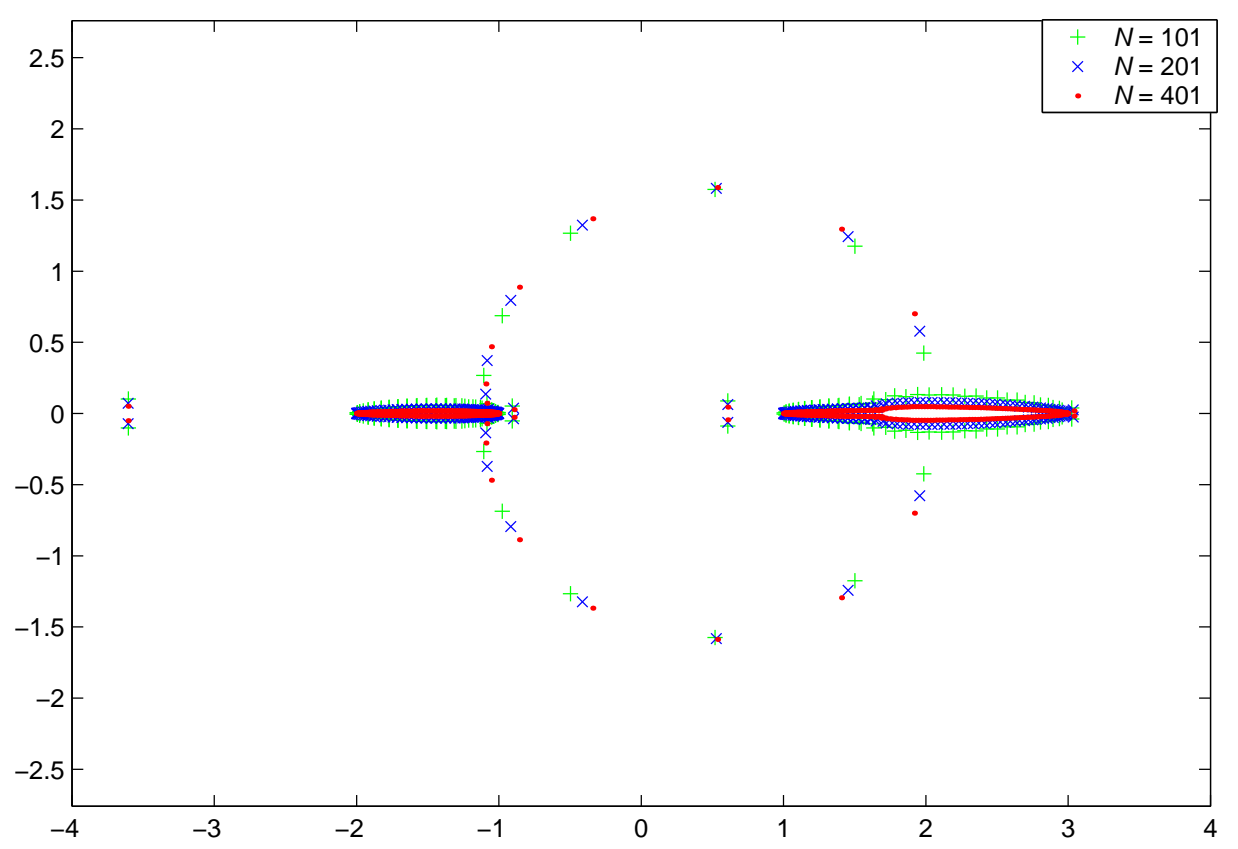

Figure 7: $\operatorname{Spec}_{2}\left(B, \mathcal{L}_{N}\right)$. 


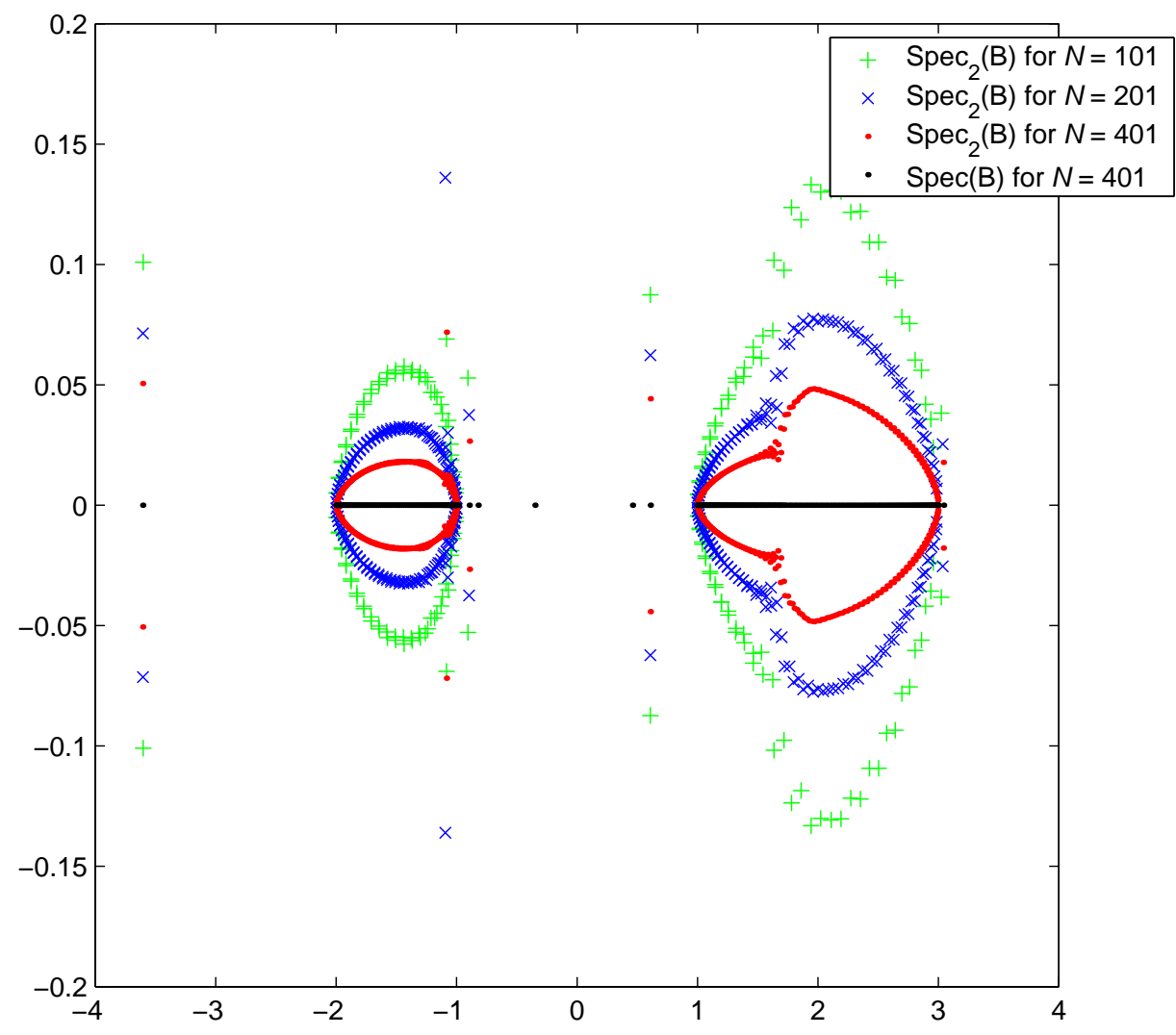

Figure 8: Detailed view of Figure 7 near the real axis and, for comparison, $\operatorname{Spec}\left(B, \mathcal{L}_{N}\right)$. 


\section{Example II: Stokes type system of ODEs}

Our next example is an operator $H$ in $L_{2}([-\pi, \pi])$ generated by a simple $2 \times 2$ Stokes type system of ODEs subject to the periodic boundary conditions,

$$
H=\left(\begin{array}{cc}
-\frac{d^{2}}{d x^{2}} & -\frac{d}{d x} \\
\frac{d}{d x} & 2
\end{array}\right)
$$

Finding the spectrum of $H$ explicitly is easy. The essential spectrum is found by using a general result on Agmon-Douglis-Nirenberg (that is, mixed order) elliptic systems GG, which gives

$$
\operatorname{Spec}_{\mathrm{ess}}(H)=\left\{\lambda \in \mathbb{C} \mid \exists \xi \in \mathbb{R} \backslash\{0\}: \operatorname{det}\left(\begin{array}{cc}
\xi^{2} & i \xi \\
-i \xi & 2-\lambda
\end{array}\right)=0\right\}=\{1\} .
$$

To find the discrete spectrum, consider the system of equations

$$
\left\{\begin{aligned}
-u^{\prime \prime}-v^{\prime} & =\lambda u \\
u^{\prime}+2 v & =\lambda v
\end{aligned}\right.
$$

subject to the periodic boundary conditions. Expressing $v$ from the second equation and substituting the result into the first one gives

$$
-u^{\prime \prime}=\frac{\lambda(\lambda-2)}{\lambda-1} u \text {. }
$$

Comparing this equation with the standard periodic spectral problem for $-d^{2} / d x^{2}$ we conclude that

$$
\lambda \in \operatorname{Spec}_{\text {disc }}(H) \quad \text { iff } \quad \frac{\lambda(\lambda-2)}{\lambda-1} \in \operatorname{Spec}\left(-\frac{d^{2}}{d x^{2}}\right)=\left\{k^{2}: k \in \mathbb{Z}\right\} .
$$

This implies that $H$ has two series of eigenvalues $\lambda_{k}^{ \pm}$given by

$$
\lambda_{k}^{ \pm}=\frac{k^{2}+2 \pm \sqrt{\left(k^{2}+2\right)^{2}-4 k^{2}}}{2}, \quad k=0,1,2, \ldots
$$

Each eigenvalue $\lambda_{k}^{-}$or $\lambda_{k}^{+}$with $k>0$ is of multiplicity 2. The eigenvalues of the "minus" series are located in the interval $[0,1)$ and converge to 1 as $k \rightarrow \infty$. The eigenvalues of the "plus" series are located in the interval 
$[2,+\infty)$ and tend to $+\infty$ as $k \rightarrow \infty$. Note that the interval $(1,2)$ is spectrumfree. The eigenvectors $(u, v)$ of $H$ corresponding to the eigenvalue $\lambda_{k}^{ \pm}, k>0$ are linear combinations of

$$
\left(\begin{array}{c}
1 \\
i k /\left(\lambda_{k}^{ \pm}-2\right)
\end{array}\right) e^{i k x} \text { and }\left(\begin{array}{c}
1 \\
-i k /\left(\lambda_{k}^{ \pm}-2\right)
\end{array}\right) e^{-i k x} .
$$

The eigenvectors corresponding to $\lambda_{0}^{-}=0$ and $\lambda_{0}^{+}=2$ are proportional to $\left(\begin{array}{l}1 \\ 0\end{array}\right)$ and $\left(\begin{array}{l}0 \\ 1\end{array}\right)$ respectively.

The projection method we use in the numerical analysis of the spectrum of $H$ is the standard finite element method (FEM). It turns out (see Section 7 below) that the results of calculations depend dramatically upon the choice of the corresponding spaces of finite elements.

We start with the simplest possible choice - the space $\mathbf{L}_{N}^{p}$ of one dimensional periodic Lagrange elements of order $p$, that is, of continuous functions which are piecewise polynomials of degree $p$. Here $N$ is the number of mesh node points on the interval $[-\pi, \pi]$, so the total dimension of $\mathbf{L}_{N}^{p}$ is $N p$.

Remark 4.1. In most of the numerical experiments, we use just the linear Lagrange elements (i.e., of order 1). We shall therefore write $\mathbf{L}_{N}:=\mathbf{L}_{N}^{1}$. Additional tests have shown that varying the order affects neither the qualitative picture, nor the rate of convergence.

It is important to note that the spaces $\mathbf{L}_{N}^{p}$ are embedded into the Sobolev space $W_{2}^{1}([-\pi, \pi])$ but not into the Sobolev spaces $W_{2}^{2}([-\pi, \pi])$. Thus, the elements of $\mathbf{L}_{N} \times \mathbf{L}_{N}$ do not necessarily belong to $\operatorname{Dom}(H)$. However, they belong to the domain of the corresponding quadratic form, which is sufficient for the FEM.

The Lagrange elements are provided by all standard FEM packages, including, e.g., FEMLAB (see [FEM]).

The calculation of $\operatorname{Spec}\left(H, \mathbf{L}_{N} \times \mathbf{L}_{N}\right)$ produces a very striking result: it gives very accurately several initial eigenvalues from the $\lambda^{ \pm}$series, filling at the same time the entire interval $(1,2)$ with spurious eigenvalues (see Figure 9, which, for brevity, shows only the eigenvalues lying in the interval $[0,6])$. In the particular case $N=100$, there are 15 eigenvalues in $[0,1], 99$ eigenvalues in $[2, \infty)$, and the remaining 86 eigenvalues are in $(1,2)$.

The subspace $\mathcal{L}=\mathbf{L}_{N} \times \mathbf{L}_{N}$ used above is unsuitable for the calculation of the second order relative spectra $\operatorname{Spec}_{2}(H, \mathcal{L})$ since for elements of this space the left-hand side of (何) does not make sense. Therefore, as usual when dealing with fourth order ODES, let us introduce the standard space of periodic cubic Hermite elements $\mathbf{M}_{N}$, whose functions are continuously 


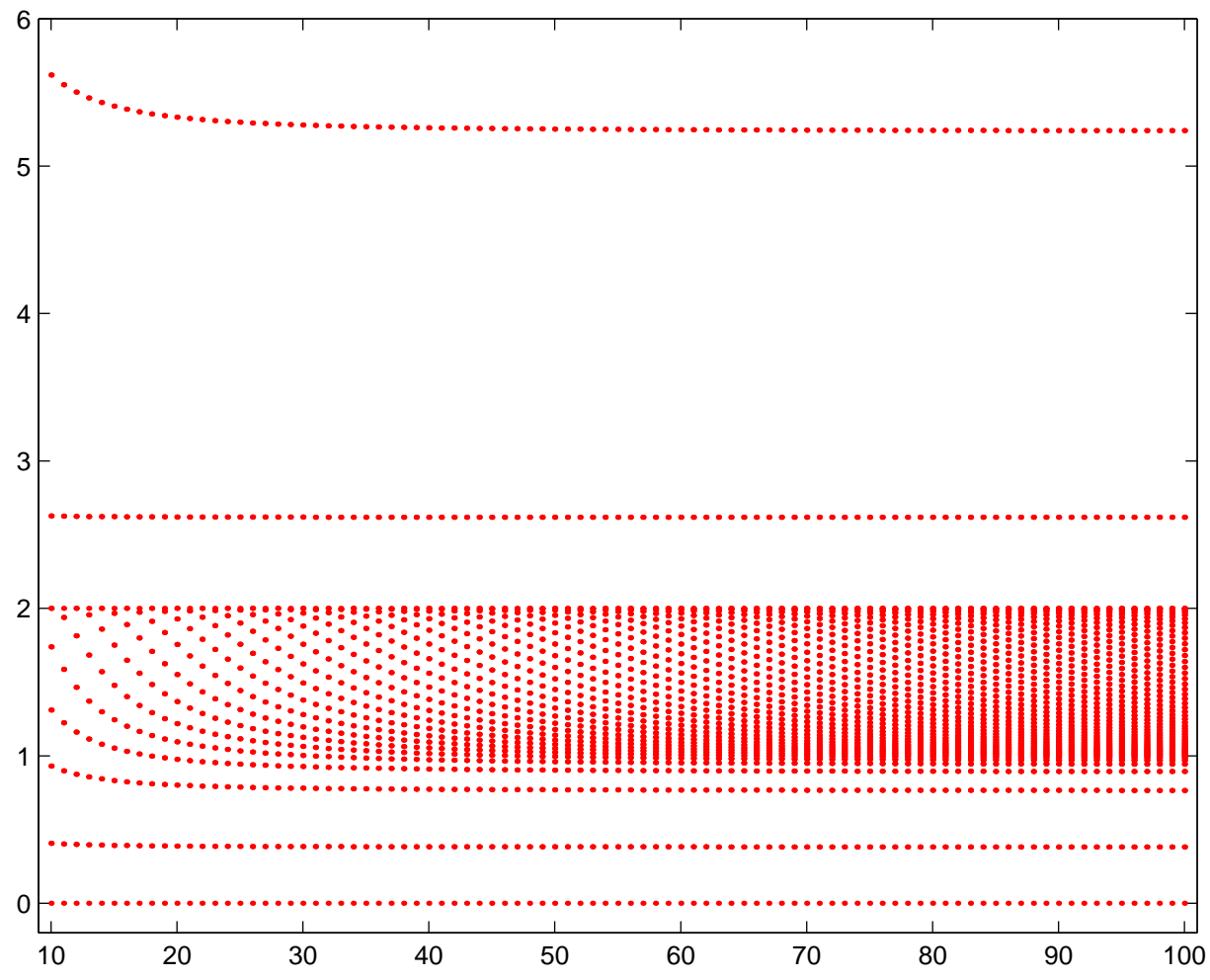

Figure 9: FEM calculated $\operatorname{Spec}\left(H, \mathbf{L}_{N} \times \mathbf{L}_{N}\right)$ plotted against $N$. All eigenvalues in the interval $(1,2)$ are spurious!

differentiable and are piecewise polynomials of degree 3 . They belong to the Sobolev space $W_{2}^{2}([-\pi, \pi])$ so they are smoother than elements of $\mathbf{L}_{N}$. Here again $N$ is the number of mesh node points, so that $\operatorname{dim} \mathbf{M}_{N}=2 N$.

Since the operator $H$ is of mixed order, it is sufficient to choose the space $\mathbf{M}_{N} \times \mathbf{L}_{N}$ for the projection space in (4). The results of calculation of $\operatorname{Spec}_{2}\left(H, \mathbf{M}_{N} \times \mathbf{L}_{N}\right)$ are shown in Figures 10 and 11. The first figure (with different scales along the real and imaginary axes) demonstrates once more the advantage of this method in eliminating the spectral pollution. Note also the series of points of $\operatorname{Spec}_{2}\left(H, \mathbf{M}_{N} \times \mathbf{L}_{N}\right)$ along the line $\operatorname{Re} \lambda=1-$ this indicates the presence of the essential spectrum of $H$ at $\lambda=1$. The second figure shows all computed points of the second order relative spectra for $N=25,50$, and 100 .

The points of the second order relative spectra give good approximations for the low eigenvalues of $H$. We postpone the discussion of the rates of convergence in various cases until Section 7 . We note only that the number of well approximated eigenvalues increases with $N$, see Figure 11 . 


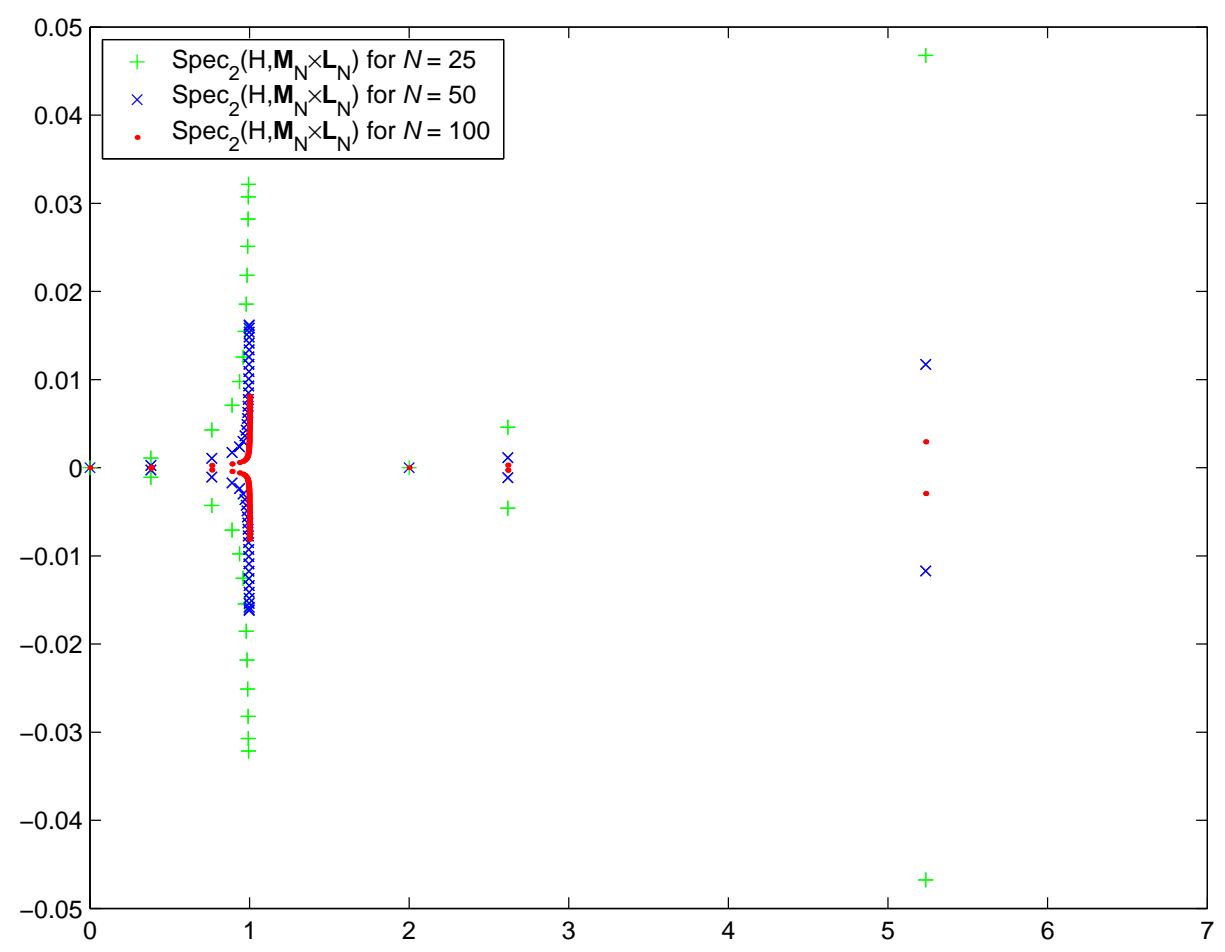

Figure 10: Second order relative $\operatorname{spectra} \operatorname{Spec}_{2}\left(H, \mathbf{M}_{N} \times \mathbf{L}_{N}\right)$ for various values of $N$ - low eigenvalues.

Examples I and II are discussed further in Sections 6 and 7; we proceed now to the proofs of the main theoretical results stated in Section 2. 


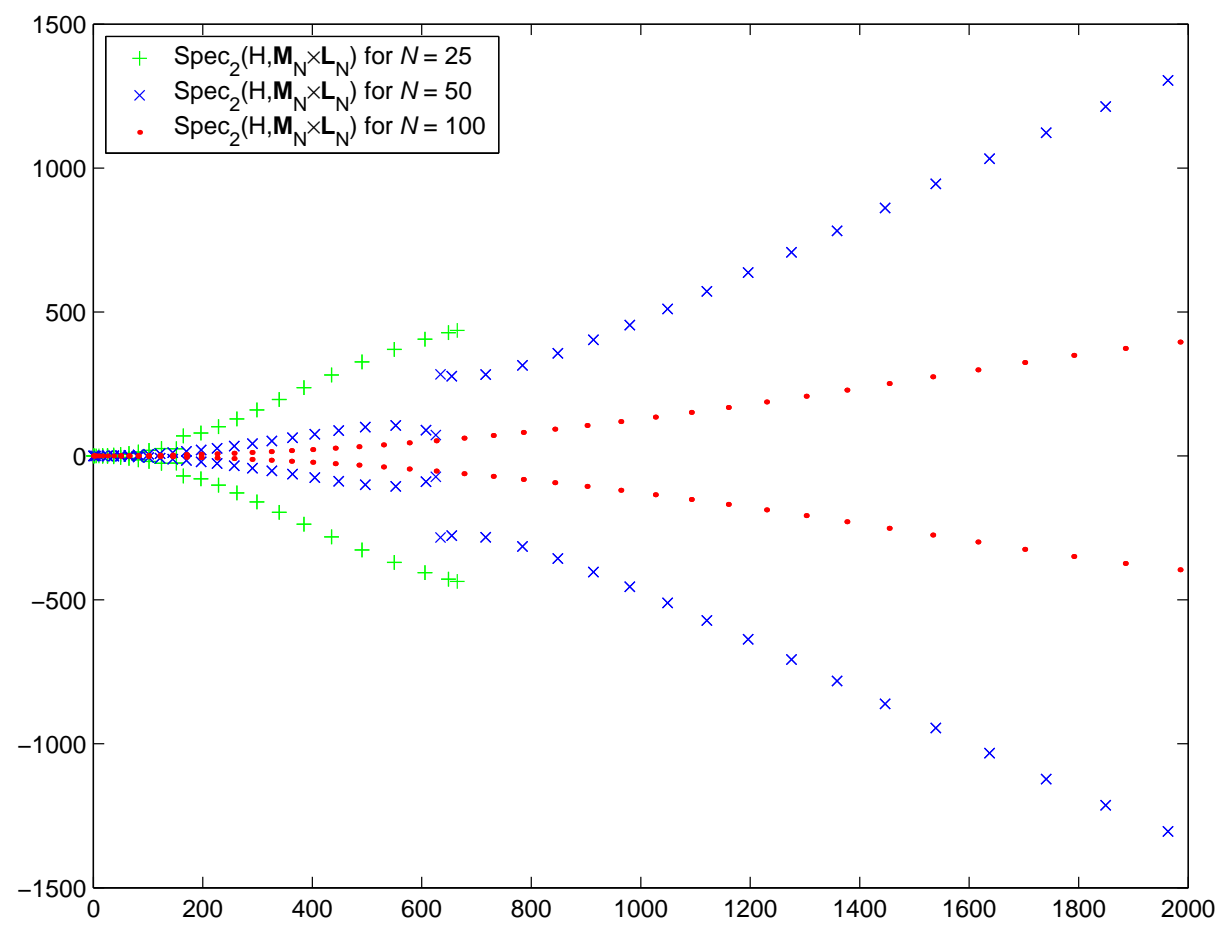

Figure 11: Second order relative $\operatorname{spectra} \operatorname{Spec}_{2}\left(H, \mathbf{M}_{N} \times \mathbf{L}_{N}\right)$ for various values of $N$ - all eigenvalues. 


\section{Proofs of the main results}

Proof of Theorem 2.1. It is clear that there exists $\lambda_{-} \in \widehat{\operatorname{Spec}_{\text {ess }}}(A)$ which lies strictly to the left of $\lambda$. Consider first the case $\lambda_{-} \in \operatorname{Spec}_{\text {ess }}(A)$. If $\lambda_{-}$is an isolated point of $\operatorname{Spec}(A)$, then it is an eigenvalue of infinite multiplicity and we define $\mathcal{H}_{j}^{-}:=\operatorname{span}\left(\left\{e_{j}\right\}\right)$, where $e_{j}, j \in \mathbb{N}$ are mutually orthogonal eigenvectors of $A$ corresponding to $\lambda_{-}$. If $\lambda_{-}$is not an isolated point, there exist pair-wise disjoint compact sets $W_{j}^{-} \subset \mathbb{R}$ lying strictly to the left of $\lambda$ and such that $W_{j}^{-} \cap \operatorname{Spec}(A) \neq \varnothing, j \in \mathbb{N}$. In this case we choose $\mathcal{H}_{j}^{-}=E\left(W_{j}^{-}\right) \mathcal{H}$, where $E(\cdot)$ are the spectral projections corresponding to $A$. Finally, if no point of $\operatorname{Spec}_{\text {ess }}(A)$ lies strictly to the left of $\lambda$, then $\lambda_{-}=-\infty$ and there exist eigenvalues $\lambda_{j}<\lambda$ of $A$ converging to $-\infty$. Here we set $\mathcal{H}_{j}^{-}:=E\left(\left\{\lambda_{j}\right\}\right)$. In all the above cases $\mathcal{H}_{j}^{-} \subset E((-\infty, \lambda)), j \in \mathbb{N}$.

Similarly one can define subspaces $\mathcal{H}_{j}^{+} \subset E((\lambda,+\infty)), j \in \mathbb{N}$. It is clear that $\mathcal{H}_{j}^{ \pm}$are all mutually orthogonal, $\mathcal{H}_{j}^{ \pm} \subset \operatorname{Dom}(A), A \mathcal{H}_{j}^{ \pm} \subset \mathcal{H}_{j}^{ \pm}$, and there exist $r_{k}>0$ such that $r_{k} \nearrow+\infty$ and $\mathcal{H}_{j}^{ \pm} \subset E\left(\left[-r_{k}, r_{k}\right]\right) \mathcal{H}, j \leq k$. Let $\mathcal{H}_{0}$ be the closed linear subspace spanned by $\mathcal{H}_{j}^{ \pm}, j \in \mathbb{N}$ and $\mathcal{H}_{0}^{\perp}$ be its orthogonal complement.

Let $v_{k}^{ \pm} \in \mathcal{H}_{k}^{ \pm},\left\|v_{k}^{ \pm}\right\|=1$ and $\mu_{k}^{ \pm}:=\left(A v_{k}^{ \pm}, v_{k}^{ \pm}\right)$. Then it follows from the spectral theorem that $\mu_{k}^{-}<\lambda<\mu_{k}^{+}, k \in \mathbb{N}$. Let $\mathcal{L}_{k}$ be the closed linear subspace spanned by $\mathcal{H}_{j}^{ \pm}, j<k, E\left(\left[-r_{k}, r_{k}\right]\right) \mathcal{H}_{0}^{\perp}$, and the vector

$$
u_{k}:=\sqrt{\frac{\mu_{k}^{+}-\lambda}{\mu_{k}^{+}-\mu_{k}^{-}}} v_{k}^{-}+\sqrt{\frac{\lambda-\mu_{k}^{-}}{\mu_{k}^{+}-\mu_{k}^{-}}} v_{k}^{+} .
$$

It is easily seen that the sequence $\left(\mathcal{L}_{k}\right)_{k \in \mathbb{N}}$ is increasing and belongs to $\Lambda(A)$. Since $v_{k}^{ \pm}, A v_{k}^{ \pm} \in \mathcal{H}_{k}^{ \pm} \subset E\left(\left[-r_{k}, r_{k}\right]\right) \mathcal{H}$, the orthogonality properties of $\mathcal{H}_{j}^{ \pm}$ imply

$$
\begin{aligned}
& \left\|u_{k}\right\|=1, \\
& \left((A-\lambda I) u_{k}, u_{k}\right)=\frac{\mu_{k}^{+}-\lambda}{\mu_{k}^{+}-\mu_{k}^{-}}\left(A v_{k}^{-}, v_{k}^{-}\right)+\frac{\lambda-\mu_{k}^{-}}{\mu_{k}^{+}-\mu_{k}^{-}}\left(A v_{k}^{+}, v_{k}^{+}\right)- \\
& \lambda\left\|u_{k}\right\|^{2}=\frac{\mu_{k}^{+}-\lambda}{\mu_{k}^{+}-\mu_{k}^{-}} \mu_{k}^{-}+\frac{\lambda-\mu_{k}^{-}}{\mu_{k}^{+}-\mu_{k}^{-}} \mu_{k}^{+}-\lambda=0, \\
& \left((A-\lambda I) u_{k}, v\right)=0, \quad \forall v \in E\left(\left[-r_{k}, r_{k}\right]\right) \mathcal{H}_{0}^{\perp} \cup \bigcup_{j<k}\left(\mathcal{H}_{j}^{-} \cup \mathcal{H}_{j}^{+}\right) .
\end{aligned}
$$

Hence $\left((A-\lambda I) u_{k}, u\right)=0, \forall u \in \mathcal{L}_{k}$, i.e. $P_{k}(A-\lambda I) u_{k}=0$, i.e. $P_{k} A u_{k}=\lambda u_{k}$, i.e. $\lambda$ is an eigenvalue of $P_{k} A: \mathcal{L}_{k} \rightarrow \mathcal{L}_{k}, \forall k \in \mathbb{N}$.

Remark 5.1. If $\mathcal{H}$ is separable, the subspaces $\mathcal{L}_{k}$ in Theorem 2.1 can be made finite dimensional. Indeed, let $e_{j, m}^{ \pm}, m=1, \ldots$ be an orthonormal 
basis of $\mathcal{H}_{j}^{ \pm}$and $e_{m}^{0}, m=1, \ldots$ be an orthonormal basis of $\mathcal{H}_{0}^{\perp}$. These bases exist because all subspaces of $\mathcal{H}$ are separable. Let $\mathcal{L}_{k}$ be the closed linear subspace spanned by $e_{j, m}^{ \pm}$and $E\left(\left[-r_{k}, r_{k}\right]\right) e_{m}^{0}$ with $j, m<k$, and the vector

$$
u_{k}:=\sqrt{\frac{\mu_{k}^{+}-\lambda}{\mu_{k}^{+}-\mu_{k}^{-}}} e_{k, 1}^{-}+\sqrt{\frac{\lambda-\mu_{k}^{-}}{\mu_{k}^{+}-\mu_{k}^{-}}} e_{k, 1}^{+} .
$$

Then it is clear that $\mathcal{L}_{k}$ is finite dimensional, and the rest of the proof of Theorem 2.1 remains unchanged.

Proof of Theorem 2.9. It is sufficient to prove that for any finite dimensional $\mathcal{L} \subset \operatorname{Dom}(A)$ and any $\varepsilon, R>0$ there exists $\mathcal{L}^{\prime} \subset \operatorname{Dom}(A)$ such that

$$
\left\|P-P^{\prime}\right\|<\varepsilon \quad \text { and } \operatorname{Spec}\left(A, \mathcal{L}^{\prime}\right) \subset(R,+\infty),
$$

where $P: \mathcal{H} \rightarrow \mathcal{L}$ and $P^{\prime}: \mathcal{H} \rightarrow \mathcal{L}^{\prime}$ are the corresponding orthogonal projections.

Let $m:=\operatorname{dim} \mathcal{L}$ and $\varphi_{1}, \ldots, \varphi_{m}$ be an orthonormal basis of $\mathcal{L}$. Since $A$ is not bounded above, for any $\tau>0$ there exist mutually orthogonal vectors $\psi_{1}, \ldots, \psi_{m} \in \operatorname{Dom}(A) \cap E((\tau,+\infty)) \mathcal{H}$ which we normalize by $\left\|\psi_{l}\right\|=\delta$, $l=1, \ldots, m$, where $\delta \in(0,1)$. The numbers $\tau$ and $\delta$ will be chosen later.

It is easy to see that the vectors $\varphi_{1}+\psi_{1}, \ldots, \varphi_{m}+\psi_{m}$ are linearly independent. Indeed, for any $c_{1}, \ldots, c_{m} \in \mathbb{C}$ Parseval's identity implies

$$
\left\|\sum_{l=1}^{m} c_{l}\left(\varphi_{l}+\psi_{l}\right)\right\| \geq\left\|\sum_{l=1}^{m} c_{l} \varphi_{l}\right\|-\left\|\sum_{l=1}^{m} c_{l} \psi_{l}\right\|=(1-\delta)\left(\sum_{l=1}^{m}\left|c_{l}\right|^{2}\right)^{1 / 2} .
$$

Similarly

$$
\left\|\sum_{l=1}^{m} c_{l}\left(\varphi_{l}+\psi_{l}\right)\right\| \leq(1+\delta)\left(\sum_{l=1}^{m}\left|c_{l}\right|^{2}\right)^{1 / 2}
$$

Let $\mathcal{L}^{\prime}$ be the subspace spanned by $\varphi_{1}+\psi_{1}, \ldots, \varphi_{m}+\psi_{m}$ and consider the operators $J: \mathcal{L} \rightarrow \mathcal{L}^{\prime}$ and $J^{\prime}: \mathcal{L}^{\prime} \rightarrow \mathcal{L}$ defined by

$$
J\left(\sum_{l=1}^{m} c_{l} \varphi_{l}\right)=\sum_{l=1}^{m} c_{l}\left(\varphi_{l}+\psi_{l}\right), \quad J^{\prime}\left(\sum_{l=1}^{m} c_{l}\left(\varphi_{l}+\psi_{l}\right)\right)=\sum_{l=1}^{m} c_{l} \varphi_{l} .
$$

It is clear that $J^{\prime} J=I_{\mathcal{L}}, J J^{\prime}=I_{\mathcal{L}^{\prime}}$ and

$$
(I-J)\left(\sum_{l=1}^{m} c_{l} \varphi_{l}\right)=-\sum_{l=1}^{m} c_{l} \psi_{l}, \quad\left(I-J^{\prime}\right)\left(\sum_{l=1}^{m} c_{l}\left(\varphi_{l}+\psi_{l}\right)\right)=\sum_{l=1}^{m} c_{l} \psi_{l} .
$$


It follows from Parseval's identity and (10), (11) that

$$
\|J\| \leq 1+\delta, \quad\left\|J^{\prime}\right\| \leq \frac{1}{1-\delta}, \quad\|(I-J) P\| \leq \delta, \quad\left\|\left(I-J^{\prime}\right) P^{\prime}\right\| \leq \frac{\delta}{1-\delta}
$$

Further,

$$
\left\|P-P^{\prime}\right\| \leq\left\|P-P^{\prime} P\right\|+\left\|P^{\prime} P-P^{\prime}\right\|=\left\|P-P^{\prime} P\right\|+\left\|P P^{\prime}-P^{\prime}\right\|,
$$

since $\left(P^{\prime} P-P^{\prime}\right)^{*}=P P^{\prime}-P^{\prime}$. Further, for any $x \in \mathcal{H}$ the closest point of $\mathcal{L}^{\prime}$ is $P^{\prime} x$. Hence $\left\|P-P^{\prime} P\right\| \leq\|P-J P\|$. Similarly $\left\|P^{\prime}-P P^{\prime}\right\| \leq\left\|P^{\prime}-J^{\prime} P^{\prime}\right\|$. Consequently

$$
\left\|P-P^{\prime}\right\| \leq\|(I-J) P\|+\left\|\left(I-J^{\prime}\right) P^{\prime}\right\| \leq \delta+\frac{\delta}{1-\delta}=\frac{\delta(2-\delta)}{1-\delta} .
$$

Fix $\delta \in(0,1)$ such that $\delta(2-\delta) /(1-\delta)<\varepsilon$. Then $\left\|P-P^{\prime}\right\|<\varepsilon$.

Since $\mathcal{L}$ is a finite dimensional subspace of $\operatorname{Dom}(A)$, there exists $M>0$ such that $\|A u\| \leq M\|u\|, \forall u \in \mathcal{L}$. Take an arbitrary

$$
v=\sum_{l=1}^{m} c_{l}\left(\varphi_{l}+\psi_{l}\right) \in \mathcal{L}^{\prime}
$$

and set

$$
\varphi:=\sum_{l=1}^{m} c_{l} \varphi_{l} \in \mathcal{L}, \quad \psi:=\sum_{l=1}^{m} c_{l} \psi_{l} \in \operatorname{Dom}(A) \cap E((\tau,+\infty)) \mathcal{H} .
$$

Then

$$
\begin{aligned}
& (A v, v)=(A \psi, \psi)+2 \operatorname{Re}(A \varphi, \psi)+(A \varphi, \varphi) \geq \\
& \tau\|\psi\|^{2}-2 M\|\varphi\|\|\psi\|-M\|\varphi\|^{2}=\left(\tau \delta^{2}-2 M \delta-M\right) \sum_{l=1}^{m}\left|c_{l}\right|^{2} .
\end{aligned}
$$

Choose $\tau$ such that $\left(\tau \delta^{2}-2 M \delta-M\right) /(1+\delta)^{2} \geq R+1$. Then

$$
(A v, v) \geq \frac{\tau \delta^{2}-2 M \delta-M}{(1+\delta)^{2}}\|v\|^{2} \geq(R+1)\|v\|^{2}
$$

(see (11)). Hence $\operatorname{Spec}\left(A, \mathcal{L}^{\prime}\right) \subset[R+1,+\infty)$.

Suppose $A$ is a bounded below self-adjoint operator. Then everything said above generalizes from $\Lambda(A)$ to $\Lambda\left(Q_{A}\right)$ (see Section 2 for the definition) with almost no changes. One only needs to modify the concluding estimates 
of the proof of Theorem 2.2 in the following way. Adding if necessary a sufficiently large positive number to the bounded below operator $A$, one can assume without loss of generality that $A$ is positive definite. Then

$$
\begin{aligned}
& Q_{A}(v, v)=Q_{A}(\psi, \psi)+2 \operatorname{Re} Q_{A}(\varphi, \psi)+Q_{A}(\varphi, \varphi) \geq \\
& Q_{A}(\psi, \psi)-2 Q_{A}(\psi, \psi)^{1 / 2} Q_{A}(\varphi, \varphi)^{1 / 2} \geq \\
& \frac{1}{2} Q_{A}(\psi, \psi)-2 Q_{A}(\varphi, \varphi) \geq \frac{\tau}{2}\|\psi\|^{2}-2 M\|\varphi\|^{2}= \\
& \frac{\tau \delta^{2}-4 M}{2} \sum_{l=1}^{m}\left|c_{l}\right|^{2} .
\end{aligned}
$$

Choose $\tau$ such that $\left(\tau \delta^{2}-4 M\right) / 2(1+\delta)^{2} \geq R+1$. Then

$$
Q_{A}(v, v) \geq \frac{\tau \delta^{2}-4 M}{2(1+\delta)^{2}}\|v\|^{2} \geq(R+1)\|v\|^{2} .
$$

Hence $\operatorname{Spec}\left(A, \mathcal{L}^{\prime}\right) \subset[R+1,+\infty)$.

Now we proceed to the proof of Theorem 2.5. For any $c_{1}, c_{2} \in \mathbb{C}$ we denote by $\mathbb{D}\left(c_{1}, c_{2}\right)$ the open disk with diameter $\left(c_{1}, c_{2}\right)$.

Lemma 5.2. Let $A$ be a self-adjoint operator on a Hilbert space $\mathcal{H}$ and let $\mathcal{L}$ be a finite dimensional subspace of $\operatorname{Dom}(A)$. Suppose $a, b \in \mathbb{R}$ are such that

$$
(a, b) \cap \operatorname{Spec}(A)=\varnothing .
$$

Then

$$
\mathbb{D}(a, b) \cap \operatorname{Spec}_{2}(A, \mathcal{L})=\varnothing .
$$

Proof of Lemma 5.9. Take an arbitrary $z \in \mathbb{D}(a, b)$. The interval $(a, b)$ is seen from $z$ at an angle $\pi / 2+\varepsilon$, where $\varepsilon \in(0, \pi / 2]$. It is clear that the set

$$
F=\left\{(\lambda-z)^{2}: \lambda \in \operatorname{Spec}(A)\right\}
$$

lies in a sector with vertex at 0 and angle $\pi-2 \varepsilon$. The distance from 0 to $F$ is obviously positive. Therefore there exist $\vartheta \in(-\pi, \pi]$ and $c>0$ such that

$$
\operatorname{Re}\left(e^{i \vartheta}(\lambda-z)^{2}\right) \geq c, \forall \lambda \in \operatorname{Spec}(A) .
$$


Now applying the spectral theorem for $A$ one obtains

$$
\begin{aligned}
& \operatorname{Re}\left(e^{i \vartheta}((A-z I) u,(A-\bar{z} I) u)\right)= \\
& \operatorname{Re}\left(e^{i \vartheta}\left(\|A u\|^{2}-2 z(A u, u)+z^{2}\|u\|^{2}\right)\right)= \\
& \operatorname{Re}\left(e^{i \vartheta} \int_{\operatorname{Spec}(A)}\left(\lambda^{2}-2 \lambda z+z^{2}\right) d(E(\lambda) u, u)\right)= \\
& \int_{\operatorname{Spec}(A)} \operatorname{Re}\left(e^{i \vartheta}(\lambda-z)^{2}\right) d(E(\lambda) u, u) \geq c \int_{\operatorname{Spec}(A)} d(E(\lambda) u, u)= \\
& c\|u\|^{2}, \quad \forall u \in \mathcal{L} .
\end{aligned}
$$

Hence $u \in \mathcal{L} \backslash\{0\}$ cannot satisfy (㺼, i.e. $z \notin \operatorname{Spec}_{2}(A, \mathcal{L})$.

Proof of Theorem 2.5. Suppose the intersection in the statement of the Theorem is empty. Then there exists $\varepsilon>0$ such that

$$
\operatorname{Spec}(A) \cap[\operatorname{Re} z-|\operatorname{Im} z|-\varepsilon, \operatorname{Re} z+|\operatorname{Im} z|+\varepsilon]=\varnothing .
$$

Hence $\mathbb{D}(\operatorname{Re} z-|\operatorname{Im} z|-\varepsilon, \operatorname{Re} z+|\operatorname{Im} z|+\varepsilon)$ does not intersect $\operatorname{Spec}_{2}(A, \mathcal{L})$ according to Lemma 5.2. On the other hand, $z$ belongs to both of these sets. The obtained contradiction proves the theorem.

\section{More on Example I}

Here we describe what happens if the function $a$ of Section 3 is slightly modified. Since none of the changes we consider below affects the second order relative spectra in any serious way, we concentrate on the standard (first order) projection method.

We have performed calculations, similar to those presented in Section 3 , for the operator $A$ corresponding to the function which is obtained from $a$ by multiplying the cosines by $0.01,0.001$, or zero. The only noticeable difference with the results in Section 3 is that this time there are more spurious eigenvalues in the gap of the essential spectrum and additional spurious eigenvalues appear, as $N$ increases, quicker than in the original example (cf. Table 1 and Remark 3.1).

The situation becomes more interesting if one moves the discontinuity of $a$ from 0 to a point $y \neq 0$ :

$$
a(x)=a_{y}(x)= \begin{cases}-\frac{3}{2}+\frac{1}{2} \cos \sqrt{5} x, & \text { for } \quad-\pi \leq x<y \\ 2+\cos \sqrt{2} x, & \text { for } \quad y \leq x<\pi\end{cases}
$$


The corresponding operator of multiplication by $a_{y}$ will be denoted $A_{y}$.

To put the results into the right perspective, we need to return to the original example $A=A_{0}$ first. We know from $\operatorname{Section} 3$ that $\operatorname{Spec}\left(A, \mathcal{L}_{N}\right)$ show remarkable stability as $N=2 n+1$ increases. The first surprise happens when one looks at $\operatorname{Spec}\left(A, \mathcal{L}_{N}\right)$ for $N=2 n$, where $\mathcal{L}_{N}:=\operatorname{span}\left(\left\{e_{k}\right\}_{k=-n}^{n-1}\right)$. These also seem to be converging, but the spurious eigenvalues differ considerably from those corresponding to $N=2 n+1$ (see Figure 12 and Table 2 and compare them with Figure 2 and Table 1).

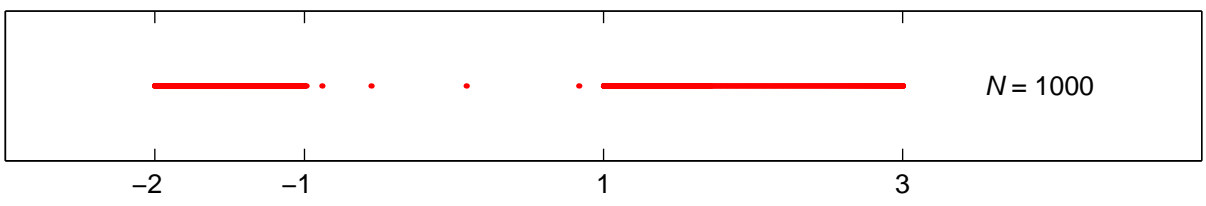

Figure 12: $\operatorname{Spec}\left(A, \mathcal{L}_{1000}\right)$.

\begin{tabular}{|c|rrrr|}
\hline$N$ & 100 & 400 & 700 & 1000 \\
\hline & & -0.9988 & -0.9935 & -0.9882 \\
$\operatorname{Spec}\left(A, \mathcal{L}_{N}\right) \cap(-1,1)$ & -0.9910 & -0.9331 & -0.9033 & -0.8832 \\
& -0.7721 & -0.6358 & -0.5853 & -0.5546 \\
& -0.0661 & 0.0317 & 0.0636 & 0.0821 \\
& 0.8883 & 0.8539 & 0.8418 & 0.8345 \\
\hline
\end{tabular}

Table 2: Eigenvalues lying in the gap of the essential spectrum of $A$ for some even values of $N$.

One might argue that this difference between the two cases occurs due to the nonsymmetric truncation in the case $N=2 n$ and that taking only odd values of $N$ is more natural. We prefer, however, to interpret this fact as 2-periodicity in $N$ of the points of $\operatorname{Spec}\left(A, \mathcal{L}_{N}\right)$ lying in the gap $(-1,1)$ of the essential spectrum of $A$, i.e., of the spurious eigenvalues of $A$. Such an interpretation is supported by Table 3, which shows the spurious eigenvalues for some consecutive values of $N$.

We show below that in a more general case of the operator $A_{y}$ the "period" depends on the location of the point $y$ of the discontinuity of $a_{y}$ (see (12)). This period is equal to 2 in the original example only because we take $y=0$ there.

Let $y=-\pi / 3$. Figure 13 shows the computed (spurious) eigenvalues of $A_{y}=a_{y} I$ in the interval $(-1,1)$ as functions of $N$. Remarkably, the 


\begin{tabular}{|c|rrrrrrrr|}
\hline$N$ & 301 & 302 & 303 & 304 & 305 & 306 & 307 & 308 \\
\hline & -0.9899 & -0.9470 & -0.9898 & -0.9467 & -0.9896 & -0.9464 & -0.9895 & -0.9461 \\
$\operatorname{Spec}\left(A, \mathcal{L}_{N}\right) \cap(-1,1)$ & -0.8468 & -0.6622 & -0.8463 & -0.6616 & -0.8458 & -0.6610 & -0.8453 & -0.6603 \\
& -0.3740 & 0.0143 & -0.3734 & 0.0147 & -0.3728 & 0.0151 & -0.3721 & 0.0155 \\
& 0.4554 & 0.8604 & 0.4555 & 0.8603 & 0.4556 & 0.8601 & 0.4557 & 0.8600 \\
\hline
\end{tabular}

Table 3: Eigenvalues lying in the gap of the essential spectrum of $A$ for some consecutive values of $N$. 2-periodicity in $N$ is clearly visible.

eigenvalues vary quite slowly with $N$ as long as $N=$ const $(\bmod 3)$. To emphasize this "3-periodicity" in $N$, we use different symbols for points with different values of $N(\bmod 3)$.

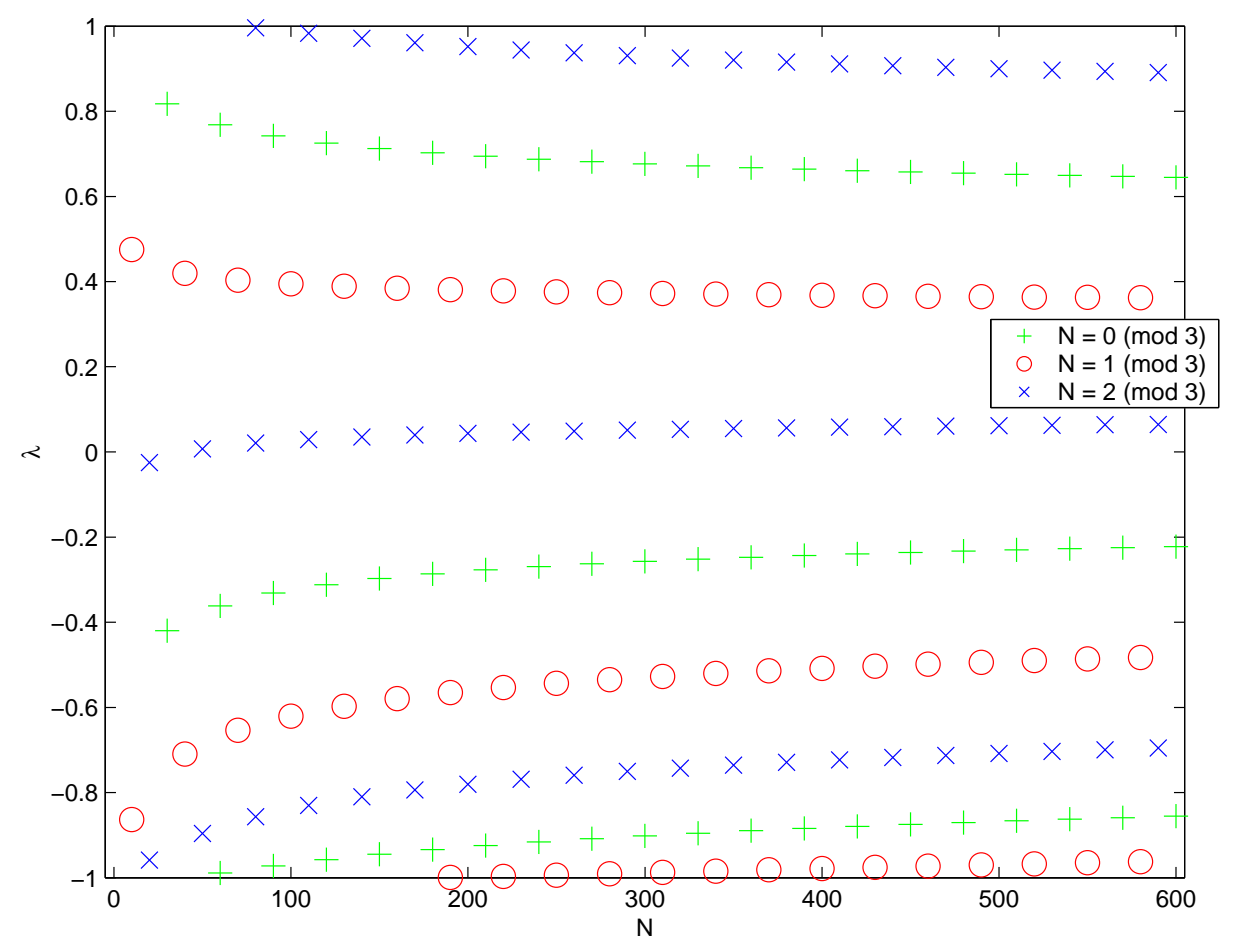

Figure 13: Points of $\operatorname{Spec}\left(A_{y}, \mathcal{L}_{N}\right), y=-\pi / 3$, in the interval $(-1,1)$ as functions of $N$.

A similar effect occurs for the operator $B_{y}$, which is obtained from the operator $B$ in (5) by replacing $a$ with $a_{y}$ (see Figure 14). However, curves corresponding to the eigenvalues in the interval $(-1,1)$, which were confirmed by the second order relative spectra as the correct ones, do not depend on the value of $N(\bmod 3)$. They also seem to converge to constants faster than 
those depending on $N(\bmod 3)$.

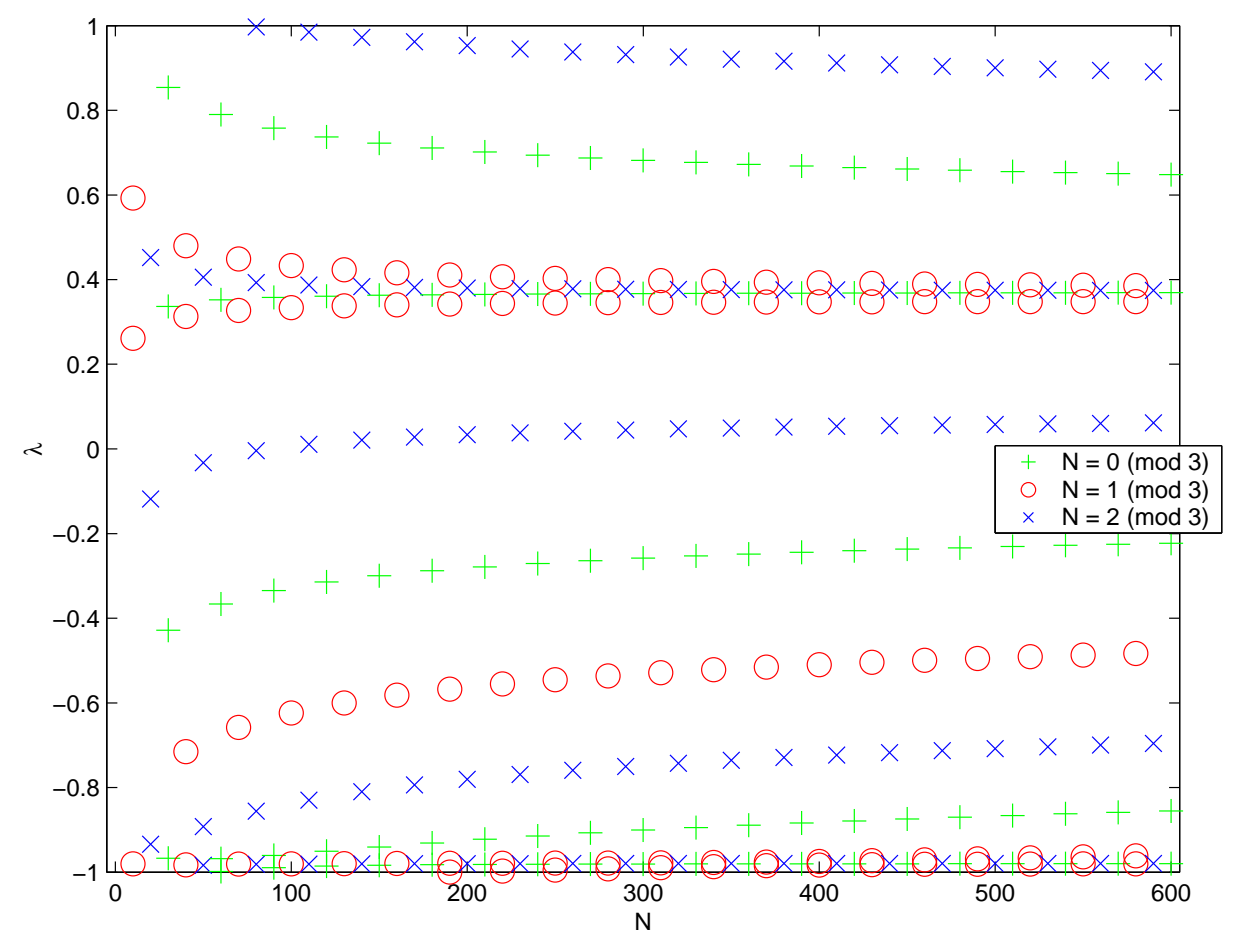

Figure 14: Points of $\operatorname{Spec}\left(B_{y}, \mathcal{L}_{N}\right), y=-\pi / 3$, in the interval $(-1,1)$ as functions of $N$. Note the presence of both "genuine" and "spurious" eigenvalues.

We have performed similar calculations with $y=-\pi /(p / q)=-\pi q / p$ for the following values $p / q=2,4,5,6,7,21,21 / 10,21 / 5,7 / 3,8 / 3$, and $5 / 2$ (see Figure 15 for the results for some of the values). In all these cases the spurious eigenvalues of $A_{y}$ showed a very clear pattern of "periodic" behaviour with the minimal "period"

$$
\omega(q / p):= \begin{cases}2, & \text { if } q=0, \\ p, & \text { if } p \text { and } q \text { are both odd } \\ 2 p, & \text { if } q \neq 0 \text { and either } p \text { or } q \text { is even. }\end{cases}
$$

Such "periodic" behaviour can be observed in Figure 15. The appearance of $\omega(q / p)$ here is probably not very surprising, as it is, for any simple fraction $q / p$, the smallest natural number such that $\omega \times($ length of $[-\pi,-\pi q / p])$ and $\omega \times($ length of $[-\pi q / p, \pi])$ are integer multiples of $2 \pi$. 

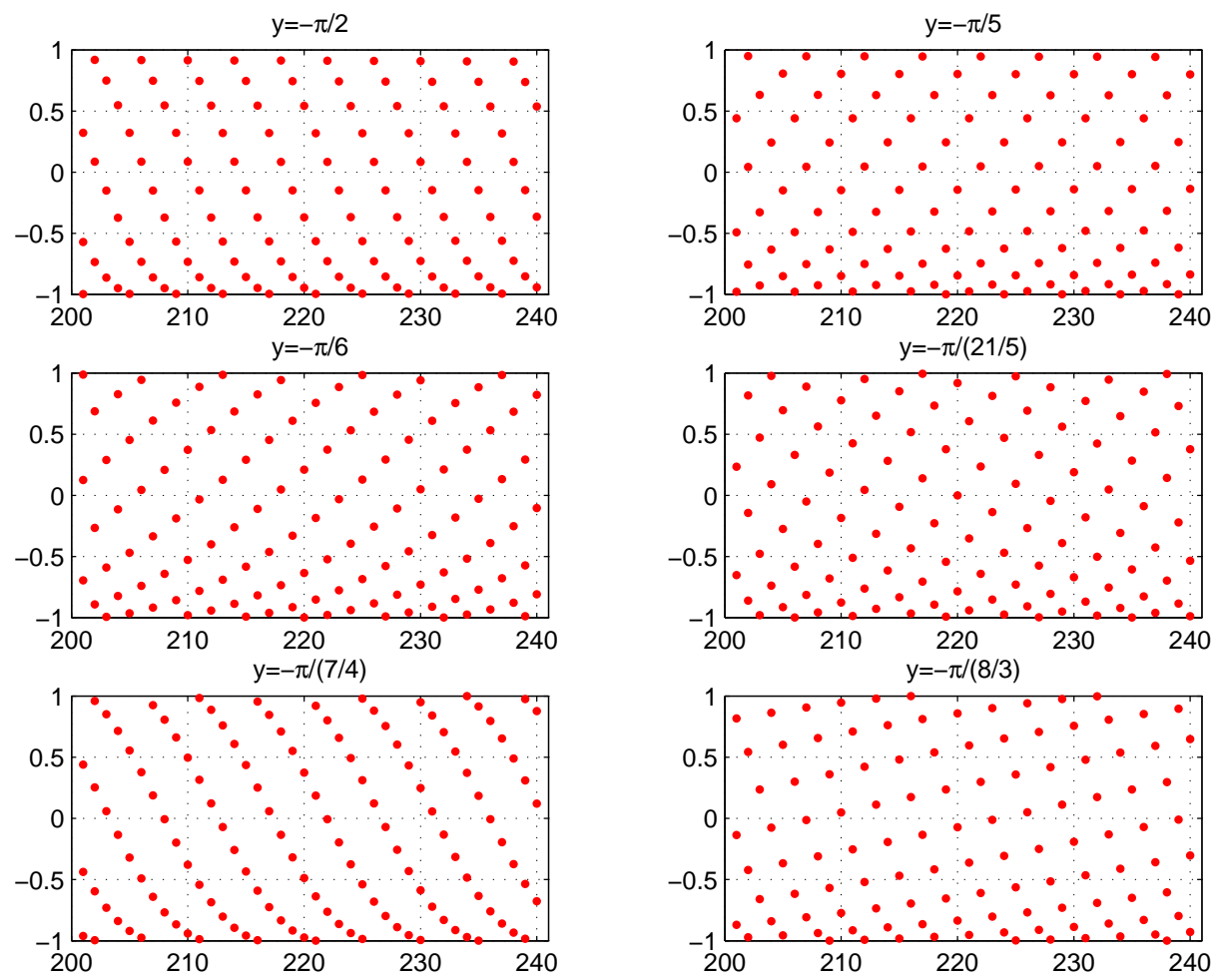

Figure 15: Points of $\operatorname{Spec}\left(A_{y}, \mathcal{L}_{N}\right)$ in the interval $(-1,1)$ as functions of $N$, plotted for various values of $y$.

\section{More on Example II}

As we have shown in Section 4, a FEM calculation of the spectrum of operator $H$ using the projection space $\mathbf{L}_{N} \times \mathbf{L}_{N}$ leads to heavy spectral pollution in the interval $(1,2)$. At the same time, the second order spectra relative to the projection space $\mathbf{M}_{N} \times \mathbf{L}_{N}$ approximate the spectrum of $H$ very well.

A natural question is whether such a discrepancy may be mostly due to the different projection spaces being used in the calculations of the first order and second order spectra. To check this, we compute the spectra $\operatorname{Spec}\left(H, \mathbf{M}_{N} \times \mathbf{L}_{N}\right)$ using the same projection spaces as we used in Section 4 for the second order relative spectra. The results (restricted to eigenvalues lying in the interval $[0,6])$ are shown in Figure 16. This time, there are no spurious eigenvalues at all! In the particular case $N=100$, we have 100 eigenvalues in $[0,1]$, and 200 eigenvalues in $[2, \infty)$ (recall that $\operatorname{dim}\left(\mathbf{M}_{N} \times\right.$ $\left.\left.\mathbf{L}_{N}\right)=3 N\right)$. The actual eigenvalues of $H$ are well approximated in this case 
as well, see Remark 7.1 below.

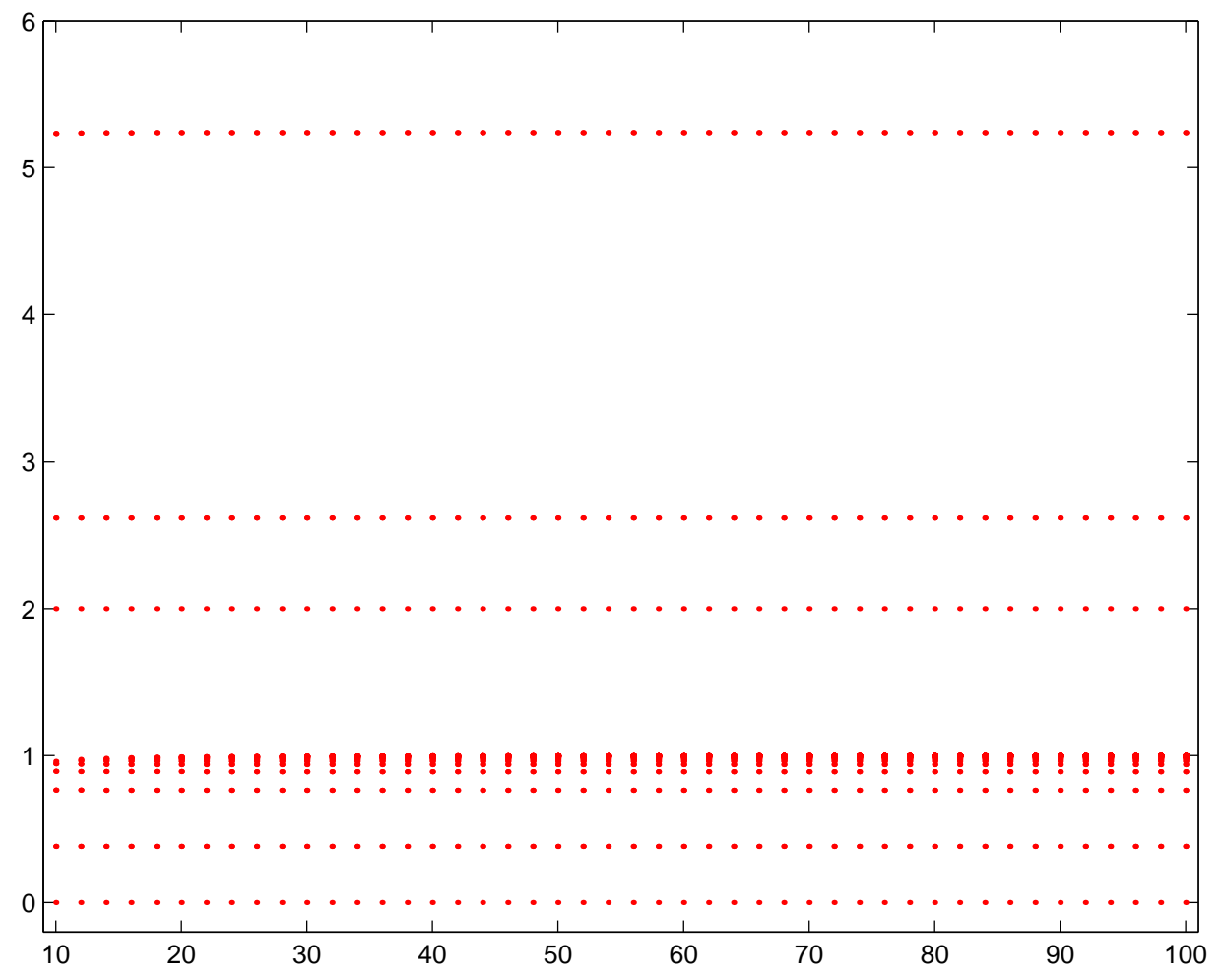

Figure 16: FEM calculated $\operatorname{Spec}\left(H, \mathbf{M}_{N} \times \mathbf{L}_{N}\right)$ plotted against $N$. There are no spurious eigenvalues!

This of course indicates that the projection spaces $\mathbf{M}_{N} \times \mathbf{L}_{N}$ are much better suited for the calculation of the spectrum of $H$ than the projection spaces $\mathbf{L}_{N} \times \mathbf{L}_{N}$. Such a dependence upon the choice of the spaces of finite elements is quite interesting, especially because it does not occur for the operator $-d^{2} / d x^{2}$ and because of the explicit relations which exist between the eigenvalues and eigenfunctions of $H$ and those of $-d^{2} / d x^{2}$, see (8) and (9).

The spaces $\mathbf{M}_{N} \times \mathbf{L}_{N}$ are well suited for the calculation of $\operatorname{Spec}(H)$ because they are subspaces of the mixed order Sobolev space $\left.W_{2}^{2}([-\pi, \pi)]\right) \times$ $\left.W_{2}^{1}([-\pi, \pi)]\right)$ and therefore reflect much better the mixed orders of differentiation in the operator $H$, see also [RSHSPV] and references therein. We attempt to explain this phenomenon in a more general setting below, see Remark 7.2 .

One may be tempted to think that the success of the second order method used in Section 4 is also only due to the correct choice of the finite elements. 
In order to check this assumption let us compare the results of calculation of the first order and second order relative spectra using the projection spaces $\mathbf{M}_{N} \times \mathbf{M}_{N}$. The results for $\operatorname{Spec}\left(H, \mathbf{M}_{N} \times \mathbf{M}_{N}\right)$ are shown in Figure 17 again, there is spectral pollution in the interval $(1,2)$.

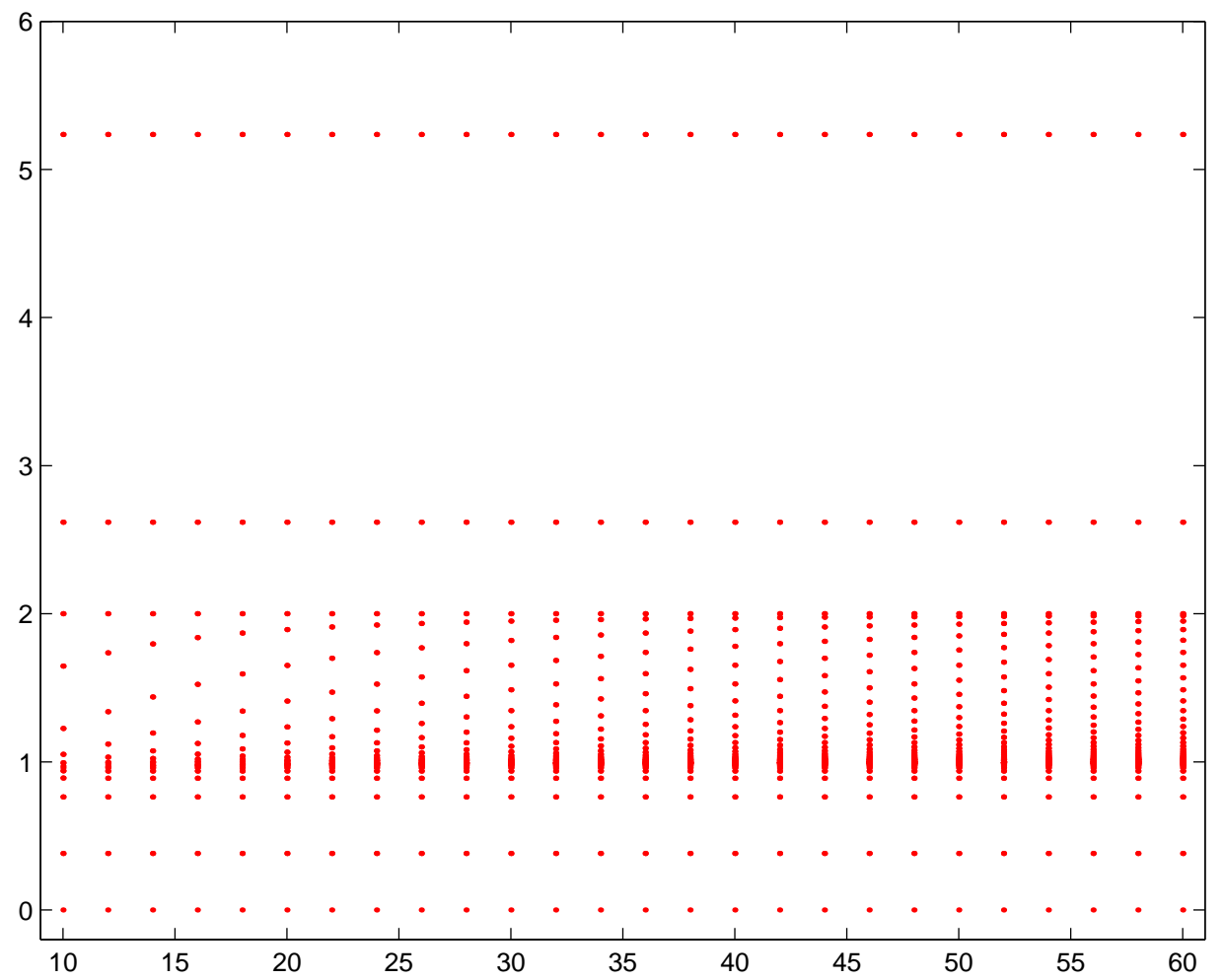

Figure 17: $\operatorname{Spec}\left(H, \mathbf{M}_{N} \times \mathbf{M}_{N}\right)$ plotted against $N$. Again, all the eigenvalues in the interval $(1,2)$ are spurious!

On the other hand, the calculations of the second order relative spectra $\operatorname{Spec}_{2}\left(H, \mathbf{M}_{N} \times \mathbf{M}_{N}\right)$ produce pictures very similar to those in Figures 10 and 11. The only noticeable difference is the presence of a number of points with relatively large moduli of imaginary parts $(\gtrsim 10$ for $N=50)$ which seem to lie on a "nice" curve passing through the point $\lambda=1$ (see Figure 18; note the different scale along the imaginary axis compared with either Figure 10 or 11).

Thus, for the spaces $\mathbf{M}_{N} \times \mathbf{M}_{N}$, the projection method using second order relative spectra works quite well, whereas the standard method again fails dramatically. 


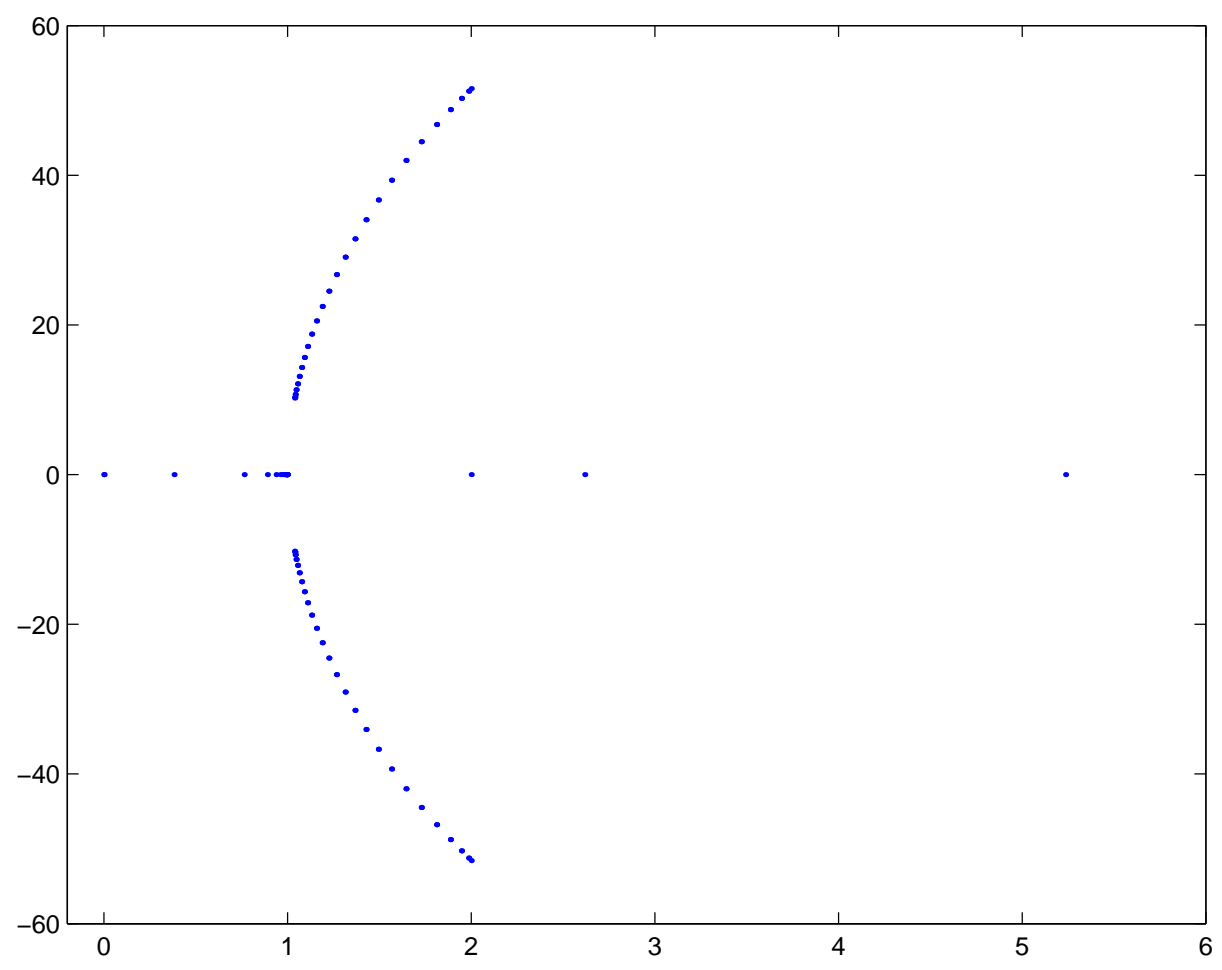

Figure 18: $\operatorname{Spec}_{2}\left(H, \mathbf{M}_{50} \times \mathbf{M}_{50}\right)$.

Remark 7.1. It should be noted that both the first order and the second order relative spectra (outside $(1,2)$ ) approximate $\operatorname{Spec}(H)$ very well, with accuracy depending on the finite elements chosen. The points of $\operatorname{Spec}\left(H, \mathbf{L}_{N} \times\right.$ $\left.\mathbf{L}_{N}\right)$, Spec $\left(H, \mathbf{M}_{N} \times \mathbf{L}_{N}\right)$, and $\operatorname{Spec}\left(H, \mathbf{M}_{N} \times \mathbf{M}_{N}\right)$ converge to the points $\lambda_{k}^{ \pm} \in \operatorname{Spec}(H)$ with the rate of about $O\left(N^{-2}\right), O\left(N^{-4}\right)$, and $O\left(N^{-6}\right)$, respectively. For the second and third choice of finite elements, the same rates of convergence are achieved by the real parts of the points of $\operatorname{Spec}_{2}\left(H, \mathbf{M}_{N} \times \mathbf{L}_{N}\right)$ and $\operatorname{Spec}_{2}\left(H, \mathbf{M}_{N} \times \mathbf{M}_{N}\right)$, whereas the imaginary parts converge to zero with the rate of about $O\left(N^{-2}\right)$ and $O\left(N^{-3}\right)$, respectively. These convergence rates were obtained by analyzing the $\log -\log$ dependence of errors upon $N$ for several low eigenvalues taken from both $\lambda^{+}$and $\lambda^{-}$series.

Remark 7.2. Here we try to explain why the standard projection method of approximating $\operatorname{Spec}(H)$ does not lead to spectral pollution in the case of the projection spaces $\mathbf{M}_{N} \times \mathbf{L}_{N}$. The main reason is that it seems, in this particular case, to "mimic" the way one finds $\operatorname{Spec}(H)$ analytically. Indeed, let $\mathcal{L}_{1}$ be a space of finite elements for $v, \mathcal{L}_{2}$ be a space of finite elements for $u$, and let $\mathcal{L}_{2}^{\prime}:=\left\{\psi^{\prime}: \psi \in \mathcal{L}_{2}\right\}$. Let $\varphi_{j}, j=1, \ldots, M$ be a basis of $\mathcal{L}_{1}$ 
and $\psi_{k}, k=1, \ldots, N$ be a basis of $\mathcal{L}_{2}$. Since the projection method does not depend on the choice of bases, provided all the calculations are precise, we can assume that the basis $\left\{\varphi_{j}\right\}$ is orthonormal. Then the finite dimensional system for (6) takes the form

$$
\left\{\begin{aligned}
\sum_{k=1}^{N}\left(\psi_{m}^{\prime}, \psi_{k}^{\prime}\right) u_{k}+\sum_{j=1}^{M}\left(\psi_{m}^{\prime}, \varphi_{j}\right) v_{j}= & \lambda \sum_{k=1}^{N}\left(\psi_{m}, \psi_{k}\right) u_{k}, \\
& m=1, \ldots, N \\
\sum_{k=1}^{N}\left(\varphi_{j}, \psi_{k}^{\prime}\right) u_{k}+2 v_{j}= & \lambda v_{j}, \\
& j=1, \ldots, M .
\end{aligned}\right.
$$

This leads to the following system for the coefficients $u_{k}$ :

$$
\begin{array}{r}
\sum_{k=1}^{N}\left(\psi_{m}^{\prime}, \psi_{k}^{\prime}\right) u_{k}+\frac{1}{\lambda-2} \sum_{k=1}^{N} \sum_{j=1}^{M}\left(\psi_{m}^{\prime}, \varphi_{j}\right)\left(\varphi_{j}, \psi_{k}^{\prime}\right) u_{k}= \\
\lambda \sum_{k=1}^{N}\left(\psi_{m}, \psi_{k}\right) u_{k}, \quad m=1, \ldots, N .
\end{array}
$$

Let $P_{1}$ be the orthogonal projection onto $\mathcal{L}_{1}$. Then

$$
P_{1} \psi_{k}^{\prime}=\sum_{j=1}^{M}\left(\psi_{k}^{\prime}, \varphi_{j}\right) \varphi_{j} \text { and }\left(P_{1} \psi_{m}^{\prime}, P_{1} \psi_{k}^{\prime}\right)=\sum_{j=1}^{M}\left(\psi_{m}^{\prime}, \varphi_{j}\right)\left(\varphi_{j}, \psi_{k}^{\prime}\right) \text {. }
$$

Hence (13) is equivalent to

$$
\begin{array}{r}
\sum_{k=1}^{N}\left(\psi_{m}^{\prime}, \psi_{k}^{\prime}\right) u_{k}+\frac{1}{\lambda-2} \sum_{k=1}^{N}\left(P_{1} \psi_{m}^{\prime}, P_{1} \psi_{k}^{\prime}\right) u_{k}=\lambda \sum_{\substack{k=1 \\
m}}^{N}\left(\psi_{m}, \psi_{k}\right) u_{k} \\
m, \ldots
\end{array}
$$

On the other hand, it is clear that (6) implies

$$
-u^{\prime \prime}-\frac{1}{\lambda-2} u^{\prime \prime}=\lambda u
$$

which is equivalent to (7). So, the projection method applied to (15) should not lead to spectral pollution. This gives the following finite dimensional system

$$
\begin{array}{r}
\sum_{k=1}^{N}\left(\psi_{m}^{\prime}, \psi_{k}^{\prime}\right) u_{k}+\frac{1}{\lambda-2} \sum_{k=1}^{N}\left(\psi_{m}^{\prime}, \psi_{k}^{\prime}\right) u_{k}=\lambda \sum_{\substack{k=1 \\
m}}^{N}\left(\psi_{m}, \psi_{k}\right) u_{k} \\
m=1, \ldots, N
\end{array}
$$

If $\mathcal{L}_{1} \supseteq \mathcal{L}_{2}^{\prime}$, then (14) and (16) coincide and the projection method applied to (6) does not pollute. If $\mathcal{L}_{1} \nsupseteq \mathcal{L}_{2}^{\prime}$, but $\mathcal{L}_{1}$ and $\mathcal{L}_{2}^{\prime}$ are "compatible", then it may well happen that (14) mimics the properties of (16) at least partially, and the projection method applied to (6) does not lead to spectral pollution. 


\section{Concluding remarks}

The main message of the paper is that projection methods using second order relative spectra may provide an efficient tool for dealing with spectral pollution. They are not much harder to implement than the standard projection methods and are likely to give useful a posteriori information (see Theorem 2.5) about the location of (at least a part of) the spectrum of a self-adjoint operator. We would feel that the paper has achieved its goal if it persuaded other people to try second order relative spectra in their problems of interest. We hope that the method we suggest here will work well in problems more serious than the model examples considered above.

We would like to stress again that we do not claim that second order relative spectra are the only way of addressing spectral pollution. In our Example I, for instance, one could argue that spectral pollution could have been detected just by looking at pictures like Figure 3, without any reference to the second order relative spectra. The argument would be that a genuine eigenfunction "cannot" look like the one in Figure 3, and hence the corresponding eigenvalue is spurious. The "periodicity" of spurious eigenvalues (see Section 6) might also have been used to detect possible spectral pollution. Indeed, those approximate eigenvalues of $B_{y}$, which were confirmed by the second order relative spectra as the correct ones, do not exhibit "periodicity" in $N$. They are present for any sufficiently large value of $N$. We have no intention to disagree with these arguments. Note however that both of these approaches are based on the results of calculations using the standard (first order) projection method, which does not see the difference between $A$ and the Toeplitz operator $T_{a}$. Consequently, they cannot distinguish between spectral pollution for the former and non-pollution for the latter. Second order relative spectra, on the other hand, are free from this problem. Our point is that it is very easy to fail to notice spectral pollution unless one is very careful, and that second order relative spectra seem to be very efficient "lie detectors".

Another perfectly legitimate argument is that one should try to avoid spectral pollution by making a sensible choice of the subspaces $\mathcal{L}_{k}$, rather than allow it to happen and then try to detect it. The standard projection method using $\mathbf{M}_{N} \times \mathbf{L}_{N}$, for instance, works very well in our Example II (see Section 7). One should, however, bear in mind that choosing "right" subspaces when applying the standard projection method in more difficult situations may prove non-trivial and require a good understanding of the problem at hand. Second order relative spectra, on the other hand, seem to provide a more robust projection method which works well even in the case of "wrong" subspaces, when the standard one fails. The case of $\mathbf{M}_{N} \times \mathbf{M}_{N}$ 
considered in Section 7 is an example of such situation.

Finally, we refer the reader to the companion paper by E. B. Davies and M. Plum [DP], where a different method of treating spectral pollution is developed.

\section{Acknowledgements}

We would like to thank Brian Davies for interest in our work and very helpful discussions. This work was partially supported by a grant from the EPSRC Spectral Theory Network.

\section{References}

[Arv] W. Arveson, $C^{*}$-algebras and numerical linear algebra, J. Functional Anal. 122, 330-360 (1994).

[BBG] D. Boffi, F. Brezzi, and L. Gastaldi, On the problem of spurious eigenvalues in the approximation of linear elliptic problems in mixed form, Math. Comp. 69, 229, 121-140 (1999).

[BDG] D. Boffi, R. G. Duran, and L. Gastaldi, A remark on spurious eigenvalues in a square, Appl. Math. Letters 12, 107-114 (1999).

[Böt] A. Böttcher, Infinite matrices and projection methods. Lectures on operator theory and its applications, Providence, RI: AMS. Fields Inst. Monogr. 3, 3-72 (1996).

[BS] A. Böttcher and B. Silbermann, Analysis of Toeplitz operators, Springer-Verlag, Berlin, 1990.

[Dav] E. B. Davies, Spectral enclosures and complex resonances for general self-adjoint operators, LMS J. Comput. Math. 1, 42-74 (1998).

[DP] E.B. Davies and M. Plum, Spectral pollution, submitted (2002).

[FEM] Femlab v.2.2 Reference Manual, Comsol AB, Stockholm, 2001; see also www. femlab.com.

[GG] G. Grubb and G. Geymonat, The essential spectrum of elliptic systems of mixed order, Math. Ann. 227, 3, 247-276 (1977). 
[HW] P. Hartman and A. Wintner, The spectra of Toeplitz's matrices, Amer. J. Math. 76, 867-882 (1954).

[Kat] T. Kato, Perturbation theory for linear operators, SpringerVerlag, Berlin, 1966.

[RSHSPV] J. Rappaz, J. Sanchez Hubert, E. Sanchez Palencia, and D. Vassiliev, On spectral pollution in the finite element approximation of thin elastic "membrane" shells, Numer. Math. 75, 473-500 (1997).

[Sha] E. Shargorodsky, Geometry of higher order relative spectra and projection methods, J. Operator Theor, 44, 43-62 (2000). 